\title{
Time Consistent Strategies for Mean-Variance Asset-Liability Management Problems
}

\author{
Hui-qiang Ma, ${ }^{1,2}$ Meng $\mathrm{Wu}^{3}$ and Nan-jing Huang ${ }^{2}$ \\ ${ }^{1}$ School of Economics, Southwest University for Nationalities, Chengdu, Sichuan 610041, China \\ ${ }^{2}$ Department of Mathematics, Sichuan University, Chengdu, Sichuan 610064, China \\ ${ }^{3}$ Business School, Sichuan University, Chengdu, Sichuan 610064, China
}

Correspondence should be addressed to Meng Wu; shancherish@hotmail.com

Received 28 May 2013; Accepted 6 August 2013

Academic Editor: Cheng Shao

Copyright (C) 2013 Hui-qiang Ma et al. This is an open access article distributed under the Creative Commons Attribution License, which permits unrestricted use, distribution, and reproduction in any medium, provided the original work is properly cited.

\begin{abstract}
This paper studies the optimal time consistent investment strategies in multiperiod asset-liability management problems under mean-variance criterion. By applying time consistent model of Chen et al. (2013) and employing dynamic programming technique, we derive two-time consistent policies for asset-liability management problems in a market with and without a riskless asset, respectively. We show that the presence of liability does affect the optimal strategy. More specifically, liability leads a parallel shift of optimal time-consistent investment policy. Moreover, for an arbitrarily risk averse investor (under the variance criterion) with liability, the time-diversification effects could be ignored in a market with a riskless asset; however, it should be considered in a market without any riskless asset.
\end{abstract}

\section{Introduction}

By using variance as a risk measure, Markowitz [1] proposed the classic mean-variance portfolio selection model, which has become the theoretical foundation of modern finance theory and has been extended in several directions. One of the main extensions for portfolio selection is to study the optimal policy in a multiperiod setting. For example, $\mathrm{Li}$ and $\mathrm{Ng}$ [2] and Zhou and Li [3] employed an embedding technique to derive analytical solutions to multiperiod and continuous-time mean-variance models, respectively. In most of the studies in the multiperiod environment, there has been a common assumption that an investor has no long-term liability. However, in reality, many investment institutions (e.g., pension funds, insurance company, and banks) have paid great attention to their portfolios while taking into account their liabilities. Further, it has been shown by Kell and Muller [4] and Sharpe and Tint [5] that liability does affect the optimal policy. More specifically, in a single-period setting, liability leads a parallel shift of meanvariance optimal investment policy and affects the meanvariance efficient frontier. Due to both theoretical interest and practical importance of asset-liability management, the research on mean-variance asset-liability management has attracted recent attentions. For example, among others, Leippold et al. [6] considered the multiperiod asset-liability management problem where the liability is exogenous and fixed and derived an analytical optimal policy and an efficient frontier. Further, they extended their research to the case where the liability is endogenous and controllable in [7]. Chiu and $\mathrm{Li}$ [8] and Xie et al. [9] studied continuous-time meanvariance asset-liability management problems, respectively. Furthermore, Xie [10] studied mean-variance model with stochastic liability in a Markovian regime switching financial market, and Zeng and Li [11] investigated asset-liability management problem in a jump diffusion market.

In most of the literature, the popular approaches of dealing with dynamic mean-variance asset-liability management problem are embedding technique which was developed by $\mathrm{Li}$ and $\mathrm{Ng}[2]$ and dual method. However, since the iteratedexpectation property does not hold for the variance operator, the optimal asset-liability management policy (called precommitment strategy) derived by both the approaches does not satisfy Bellman's optimality principle and is time inconsistent. The main reason is that, the precommitment strategy, for time interval $[t, T]$, computed at time $t$ will not necessarily 
coincide with the strategy which computed at time $t+\Delta t$. As a result, at time $t+\Delta t$, the strategy computed at time $t$ will not be implemented by the investor, unless there exists some commitment mechanism. Strotz [12] first formalized time inconsistency and pointed out that the conflict could be solved by a time-consistent strategy. Very recently, much more scholars have paid their attentions on constructing a time-consistent mean-variance portfolio choice. Among others, Basak and Chabakauri [13] provided a fully analytical characterization of the optimal time-consistent meanvariance portfolio within a general incomplete market economy. Wang and Forsyth [14] developed a numerical scheme for determining the optimal asset allocation strategy for timeconsistent, continuous time, mean-variance optimization. By allowing the trade-off between the mean and the variance of the terminal wealth varying over time, Cui et al. [15] proposed a weak time consistency to compare with Bellman's optimality principle and derived an optimal mean-variance portfolio strategy. In all the literature mentioned above, the studies mainly referred to Bellman's optimality principle. However, the requirement (for short, REQ) that local optimum is also globally optimum is not necessarily satisfied, which is an essential requirement in solving the relevant optimal portfolio problem by the dynamic programming technique. In order to make up this shortfall, Chen et al. [16], by using a time consistent dynamic risk measure, proposed a separable dynamic mean-variance model and showed that the relevant optimal investment policy satisfies not only the Bellman's optimality principle but also the REQ.

Although asset-liability management is an important issue in modern finance theory, the time-consistent assetliability management problem has not attracted enough attention. Recently, Li et al. [17] reported the time-consistent asset-liability management problem in the continuous-time setting. They employed Basak and Chabakauri's [13] model to study the continuous-time asset-liability management problem. They derived the time-consistent optimal strategy and showed that the time-consistent efficient frontier with liability is below that without liability. However, the derived time-consistent policy does not satisfy the REQ. As the aforementioned importance of the REQ, in this paper, we employ the model of Chen et al. [16] to analysis the multiperiod assetliability management problem. We derive time-consistent optimal investment policies in a market with and without a riskless asset, respectively. After comparing the optimal time-consistent policies with myopic strategies, we show that, for an arbitrarily risk averse investor, if there is a riskless asset in the market, the time-diversification effects arising from multiperiod optimization can be ignored, otherwise, the effects should be considered.

This paper proceeds as follows. In the next section, we formulate a time-consistent asset-liability management model. In Section 3, we derive the time-consistent optimal policy for a market without riskless asset. Section 4 derives the time-consistent optimal policy for a market with both riskless and risky assets. Section 5 performs numerical examples to illustrate our results. The paper is concluded in Section 6.

\section{Model Formulation}

Throughout this paper, we assume that $T \in \mathbb{N}$ is a fixed and finite time horizon and trading only takes place at time $k=$ $0,1, \ldots, T$. Let $(\Omega, \mathscr{F}, p)$ be a probability space, and let a $\sigma$ field $\mathscr{F}_{t}$ be the available information at time $t$.

Consider a security market consisting of one riskless asset and $n$ risky assets. The return of the riskless asset at the $t$ th investment period is assumed to be $s_{t}$. The return of the $i$ th risky asset at the $t$ th investment period is denoted by $R_{t}^{i}$, and the relevant random return vector is denoted by $R_{t}=$ $\left(R_{t}^{1}, \ldots, R_{t}^{n}\right)^{\prime}$ which is $\mathscr{F}_{t+1}$-measurable.

Consider an investor with an initial endowment $x_{0}$ and a liability $l_{0}$. We assume that the liability cannot be controlled and denote by $l_{t}$ the accumulative liability at time $t$. Let $Q_{t}$ be the return of the liability at the $t$ th investment period which is $\mathscr{F}_{t+1}$-measurable. It is clear that $l_{t+1}=Q_{t} l_{t}$. Assume that $\left(R_{t}^{\prime}, Q_{t}\right)^{\prime}, t=0, \ldots, T-1$, are statistically independent. Denote the expected return vector by $\mu_{t}=E\left(R_{t}\right)$ and the variancecovariance matrix by $\Sigma_{t}=\left(\operatorname{Cov}\left(R_{t}^{i}, R_{t}^{j}\right)\right)_{n \times n}$ which is assumed to be positive definite throughout this paper. It is clear that $E\left(R_{t} R_{t}^{\prime}\right)=\Sigma_{t}+\mu_{t} \mu_{t}^{\prime}$ is also positive definite. Denote by $\Sigma_{t}^{0}=$ $\left(\operatorname{Cov}\left(Q_{t}, R_{t}^{1}\right), \ldots, \operatorname{Cov}\left(Q_{t}, R_{t}^{n}\right)\right)^{\prime}$ the covariance vector. The investor begins his/her investment at time 0 and invests the cash amount $u_{t}^{i}$ in the $i$ th risky asset at the beginning of the $t$ th investment period, where $t=0, \ldots, T-1 . u_{t}=\left(u_{t}^{1}, \ldots, u_{t}^{n}\right)^{\prime}$ is called an investment decision at the $t$ th investment period and $u=\left(u_{0}, \ldots, u_{T-1}\right)$ is an investment policy during the entire investment horizon. We denote the wealth and the surplus of the investor at time $t$ by $x_{t}$ and $S_{t}=x_{t}-l_{t}$, respectively.

A single-period conditional risk mapping is defined as

$$
\rho_{t}\left(S_{t+1} \mid \mathscr{F}_{t}\right)=\operatorname{Var}_{t}\left(S_{t+1}\right)-\lambda_{t} E_{t}\left(S_{t+1}\right),
$$

where $E_{t}\left(S_{t+1}\right)=E\left(S_{t+1} \mid \mathscr{F}_{t}\right)$ and $\operatorname{Var}_{t}\left(S_{t+1}\right)=\operatorname{Var}\left(S_{t+1} \mid\right.$ $\left.\mathscr{F}_{t}\right)$ are the single-period conditional expectation of $S_{t+1}$ and its conditional variance, respectively. Tradeoff parameter $\lambda_{t}$ as a constant, which is defined on $[0, \infty]$, is a weight which presents the relative importance of expected profit compared to the risk. Note that, if $\lambda_{t}=0$, then the investor is arbitrary risk averse who only focus on the risk; if $\lambda_{t}=\infty$, then the investor is risk neutral who only concerns maximizing their expected profit; if $\lambda_{t} \in(0, \infty)$, the investor is risk averse who considers both expected profit and the risk in his decision.

In multiperiod portfolio selection, two main optimal investment policies are the myopic and time-consistent strategies. Myopic Strategy is a strategy whereby at each time $t$ the investor determines their optimal investment decision assuming the instantaneous moments of assets returns will remain fixed at their current values for the remainder of the investment horizon. More specifically, for any $t \in\{0, \ldots, T-$ $1\}$, the myopic strategy $u_{t}^{m y}$ is a solution to the following problem:

$$
\min _{u_{t} \in \Pi_{t}} \operatorname{Var}_{t}\left(S_{t+1}\right)-\lambda_{t} E_{t}\left(S_{t+1}\right),
$$

where $\Pi_{t}$ is a set of all permit policies at time $t$. Intuitively, a myopic investor only cares about the mean and variance of the surplus at the current period. 
To measure the total risk of an investor among multiperiods (after time $t$ ), we employ a separable expected conditional mapping which is defined as (see Chen et al. [16])

$$
\sum_{j=t+1}^{T} E_{t}\left(\rho_{j-1}\left(S_{j} \mid \mathscr{F}_{j-1}\right)\right),
$$

which reflects all the risk in the future. Following this assumption, a separable dynamic mean-variance problem is defined as

$$
\min _{u \in \Pi} \sum_{j=1}^{T} E\left(\rho_{j-1}\left(S_{j} \mid \mathscr{F}_{j-1}\right)\right),
$$

where $\Pi$ is a set of all permit policies. The optimal policy of problem (4), which satisfies both Bellman's optimality principle and requirement REQ, is called Time-Consistent Strategy. Note that both Bellman's optimality principle and requirement REQ could be proved by following the methodology of Chen et al. [16]. Thus, problem (4) can be recursively solved by the dynamic programming technique. Applying the iterated-expectation property of the expected operator, that is, $E\left(E\left(\cdot \mid \mathscr{F}_{j}\right) \mid \mathscr{F}_{k}\right)=E\left(\cdot \mid \mathscr{F}_{k}\right)$ for $j>k$, we have

$$
\begin{aligned}
& \sum_{j=1}^{T} E\left(\rho_{j-1}\left(S_{j} \mid \mathscr{F}_{j-1}\right)\right) \\
& \begin{aligned}
=\rho_{0}\left(S_{1}\right)+E\left(\rho_{1}\left(S_{2}\right)\right. & \\
& +E_{1}\left(\rho_{2}\left(S_{3}\right)+\cdots\right. \\
& +E_{T-3}\left(\rho_{T-2}\left(S_{T-1}\right)\right. \\
& \left.\left.\left.+E_{T-2}\left(\rho_{T-1}\left(S_{T}\right)\right)\right) \ldots\right)\right)
\end{aligned}
\end{aligned}
$$

(for more details see Chen et al. [16]). Then, problem (4) is equivalent to

$$
\begin{aligned}
\min _{u \in \Pi} \rho_{0}\left(S_{1}\right) & \\
+E\left(\rho_{1}\left(S_{2}\right)+E_{1}\left(\rho_{2}\left(S_{3}\right)\right.\right. & \\
& +\cdots+E_{T-3}\left(\rho_{T-2}\left(S_{T-1}\right)\right. \\
& \left.\left.\left.+E_{T-2}\left(\rho_{T-1}\left(S_{T}\right)\right)\right) \ldots\right)\right) .
\end{aligned}
$$

It follows from Bellman's optimality principle and (6) that (4) is equivalent to find an optimal strategy to satisfy the following problem:

$$
\begin{aligned}
& \min _{u_{0}}\left(\rho_{0}\left(S_{1}\right)\right. \\
& +E \min _{u_{1}}\left(\rho_{1}\left(S_{2}\right)\right. \\
& +E_{1} \min _{u_{2}}\left(\rho_{2}\left(S_{3}\right)+\cdots\right. \\
& +E_{T-3} \min _{u_{T-2}}\left(\rho_{T-2}\left(S_{T-1}\right)\right. \\
& \left.\left.\left.\left.\quad+E_{T-2} \min _{u_{T-1}}\left(\rho_{T-1}\left(S_{T}\right)\right)\right) \ldots\right)\right)\right) .
\end{aligned}
$$

We solve this problem in the following sections. In order to discuss the impact of riskless asset, the market is considered in two cases: with and without riskless asset. We demonstrate these results in Sections 3 and 4, respectively.

\section{Time Consistent Optimal Strategy without Riskless Asset}

Consider a market consisting of only $n$ risky assets and assume that the wealth process $x_{t}$ is in a self-financing fashion. We list the notations of this section in Table 1. The wealth process $x_{t}$ could be described as follows:

$$
x_{t+1}=R_{t}^{\prime} u_{t}, \quad I^{\prime} u_{t}=x_{t}, \quad t=0,1, \ldots, T-1,
$$

where $I=(1, \ldots, 1)^{\prime} \in \mathbb{R}^{n}$. In this setting, problem (6) can be written as follows:

$$
\begin{aligned}
& \min \rho_{0}\left(S_{1}\right)+E_{0}\left(\rho_{1}\left(S_{2}\right)\right. \\
& +E_{1}\left(\rho_{2}\left(S_{3}\right)+\cdots\right. \\
& +E_{T-3}\left(\rho_{T-2}\left(S_{T-1}\right)\right. \\
& \left.\left.\left.+E_{T-2}\left(\rho_{T-1}\left(S_{T}\right)\right)\right) \ldots .\right)\right) \\
& \text { s.t. } \quad R_{t}^{\prime} u_{t}=x_{t+1} \text {, } \\
& I^{\prime} u_{t}=x_{t} \\
& l_{t+1}=Q_{t} l_{t} \text {, } \\
& S_{t+1}=x_{t+1}-l_{t+1}, \quad t=0,1, \ldots, T-1 \text {. }
\end{aligned}
$$

Since $\Sigma_{T-1}^{1}$ is positive definite, we have $a_{T-1}>0$. Further, it follows from the nonnegative definiteness of $E\left(R_{T-1} R_{T-1}^{\prime}\right)$ that $\Sigma_{T-2}^{1}$ is also positive definite and $a_{T-2}>0$. By using mathematical induction, we conclude that, for any $t=$ $0, \ldots, T-1, \Sigma_{t}^{1}$ is also positive definite and $a_{t}>0$.

By applying Bellman's optimality principle, the timeconsistent optimal investment policy of problem (9) is given in the following theorem.

Theorem 1. The time-consistent optimal investment policy of problem (9) is given by

$$
\begin{array}{r}
u_{t}^{*}=\frac{\left(\Sigma_{t}^{1}\right)^{-1} I}{a_{t}} x_{t}+\frac{\lambda_{t}^{1}}{2}\left(\Sigma_{t}^{1}\right)^{-1}\left(\mu_{t}-q_{t} I\right) \\
+\left(\Sigma_{t}^{1}\right)^{-1}\left(\Sigma_{t}^{0}-\widetilde{\gamma}_{t} I+\widetilde{\gamma}_{t+1} E\left(Q_{t} R_{t}\right)\right) l_{t}, \\
\text { for } t=0, \ldots, T-1 .
\end{array}
$$


TABLE 1: Model notations of Section 3.

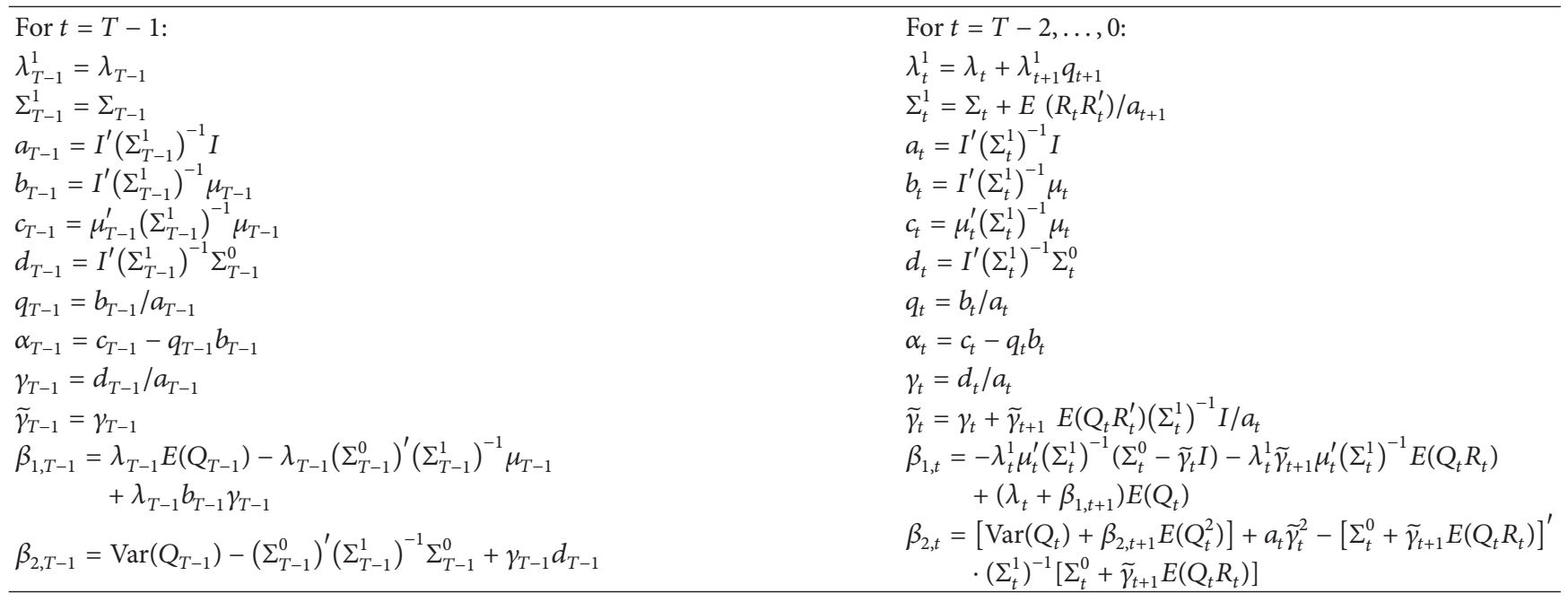

Proof. When $t=T-1$, for given wealth $x_{T-1}$ and liability $l_{T-1}$ at the beginning of the (T - 1)th period, problem (9) can be expressed as follows:

$$
\begin{array}{ll}
\min & \operatorname{Var}_{T-1}\left(S_{T}\right)-\lambda_{T-1} E_{T-1}\left(S_{T}\right) \\
\text { s.t. } & R_{T-1}^{\prime} u_{T-1}=x_{T}, \\
& I^{\prime} u_{T-1}=x_{T-1}, \\
& l_{T}=Q_{T-1} l_{T-1}, \\
& S_{T}=x_{T}-l_{T} .
\end{array}
$$

Substituting the binding constraints into the objective function, we have

$$
\begin{aligned}
\min \quad V_{T-1} & \left(x_{T-1}, l_{T-1}\right) \\
:= & u_{T-1}^{\prime} \Sigma_{T-1}^{1} u_{T-1} \\
& -2\left[\left(\Sigma_{T-1}^{0}\right)^{\prime} l_{T-1}+\frac{\lambda_{T-1}}{2} u_{T-1}^{\prime}\right] u_{T-1} \\
& +\operatorname{Var}\left(Q_{T-1}\right) l_{T-1}^{2}+\lambda_{T-1} E\left(Q_{T-1}\right) l_{T-1}
\end{aligned}
$$

s.t. $\quad I^{\prime} u_{T-1}=x_{T-1}$,

which is a linear-quadratic program. By using the Lagrange multiplier technique and letting $\omega_{T}$ be the Lagrange multiplier, the Lagrange function is defined as

$$
\begin{aligned}
L\left(u_{T-1}\right):= & u_{T-1}^{\prime} \Sigma_{T-1}^{1} u_{T-1} \\
& -2\left[\left(\Sigma_{T-1}^{0}\right)^{\prime} l_{T-1}+\frac{\lambda_{T-1}}{2} \mu_{T-1}^{\prime}\right] u_{T-1} \\
& +\operatorname{Var}\left(Q_{T-1}\right) l_{T-1}^{2}+\lambda_{T-1} E\left(Q_{T-1}\right) l_{T-1} \\
& -\omega_{T}\left(x_{T-1}-I^{\prime} u_{T-1}\right) .
\end{aligned}
$$

By using the first-order necessary optimality condition, we have

$$
\begin{gathered}
2 \Sigma_{T-1}^{1} u_{T-1}-2\left(\Sigma_{T-1}^{0} l_{T-1}+\frac{\lambda_{T-1}}{2} \mu_{T-1}\right)+\omega_{T} I=0, \\
x_{T-1}-I^{\prime} u_{T-1}=0 .
\end{gathered}
$$

From (14), we can easily have

$$
\begin{aligned}
& u_{T-1}^{*}=\left(\Sigma_{T-1}^{1}\right)^{-1}\left(\Sigma_{T-1}^{0} l_{T-1}+\frac{\lambda_{T-1}}{2} \mu_{T-1}\right)-\frac{1}{2} \omega_{T}\left(\Sigma_{T-1}^{1}\right)^{-1} I, \\
& I^{\prime} u_{T-1}^{*}= I^{\prime}\left(\Sigma_{T-1}^{1}\right)^{-1}\left(\Sigma_{T-1}^{0} l_{T-1}+\frac{\lambda_{T-1}}{2} \mu_{T-1}\right) \\
&-\frac{1}{2} \omega_{T} I^{\prime}\left(\Sigma_{T-1}^{1}\right)^{-1} I \\
&= d_{T-1} l_{T-1}+\frac{\lambda_{T-1}}{2} b_{T-1}-\frac{1}{2} a_{T-1} \omega_{T} \\
&= x_{T-1},
\end{aligned}
$$

which implies that the Lagrange multiplier $\omega_{T}$ is

$$
\begin{aligned}
\omega_{T} & =2 \frac{d_{T-1}}{a_{T-1}} l_{T-1}+\lambda_{T-1} \frac{b_{T-1}}{a_{T-1}}-2 \frac{1}{a_{T-1}} x_{T-1} \\
& =2 \gamma_{T-1} l_{T-1}+\lambda_{T-1} q_{T-1}-2 \frac{1}{a_{T-1}} x_{T-1} .
\end{aligned}
$$

Substituting $\omega_{T}$ into (16), we have

$$
\begin{aligned}
u_{T-1}^{*}= & \frac{\left(\Sigma_{T-1}^{1}\right)^{-1} I}{a_{T-1}} x_{T-1}+\left(\Sigma_{T-1}^{1}\right)^{-1}\left[\Sigma_{T-1}^{0}-\gamma_{T-1} I\right] l_{T-1} \\
& +\frac{\lambda_{T-1}}{2}\left(\Sigma_{T-1}^{1}\right)^{-1}\left[\mu_{T-1}-q_{T-1} I\right] .
\end{aligned}
$$


Further, by substituting $u_{T-1}^{*}$ into the objective function of problem (12) (see Appendix A for more details), we have

$$
\begin{aligned}
V_{T-1}^{*}\left(x_{T-1}, l_{T-1}\right)= & \frac{1}{a_{T-1}} x_{T-1}^{2}-\lambda_{T-1} q_{T-1} x_{T-1} \\
& -2 \gamma_{T-1} x_{T-1} l_{T-1}+\beta_{1, T-1} l_{T-1} \\
& +\beta_{2, T-1} l_{T-1}^{2}-\frac{\left(\lambda_{T-1}\right)^{2}}{4} \alpha_{T-1} .
\end{aligned}
$$

When $t=T-2$, for given wealth $x_{T-2}$ and liability $l_{T-2}$ at the beginning of the $(T-2)$ th period, the corresponding optimal investment problem is given as follows:

$$
\begin{array}{ll}
\min \quad & \operatorname{Var}_{T-2}\left(S_{T-1}\right)-\lambda_{T-2} E_{T-2}\left(S_{T-1}\right) \\
& +E_{T-2}\left(V_{T-1}^{*}\left(x_{T-1}, l_{T-1}\right)\right) \\
\text { s.t. } \quad & R_{T-2}^{\prime} u_{T-2}=x_{T-1}, \\
& I^{\prime} u_{T-2}=x_{T-2}, \\
& l_{T-1}=Q_{T-2} l_{T-2}, \\
& S_{T-1}=x_{T-1}-l_{T-1} .
\end{array}
$$

It follows from (20) that

$$
\begin{aligned}
E_{T-2}( & \left.V_{T-1}^{*}\left(x_{T-1}, l_{T-1}\right)\right) \\
= & \frac{1}{a_{T-1}} E_{T-2}\left(x_{T-1}^{2}\right)-\lambda_{T-1} q_{T-1} E_{T-2}\left(x_{T-1}\right) \\
& \quad-2 \gamma_{T-1} E_{T-2}\left(x_{T-1} l_{T-1}\right)+\beta_{1, T-1} E_{T-2}\left(l_{T-1}\right) \\
& +\beta_{2, T-1} E_{T-2}\left(l_{T-1}^{2}\right)-\frac{\left(\lambda_{T-1}\right)^{2}}{4} \alpha_{T-1} .
\end{aligned}
$$

Substituting $E_{T-2}\left(V_{T-1}^{*}\left(x_{T-1}, l_{T-1}\right)\right)$ and the binding constraints into the objective function of problem (21), we have

$$
\begin{array}{ll}
\min & V_{T-2}\left(x_{T-2}, l_{T-2}\right) \\
\text { s.t. } & I^{\prime} u_{T-2}=x_{T-2},
\end{array}
$$

where

$$
\begin{aligned}
V_{T-2}\left(x_{T-2}, l_{T-2}\right):= & u_{T-2}^{\prime} \Sigma_{T-2}^{1} u_{T-2} \\
& -2\left\{\left[\left(\Sigma_{T-2}^{0}\right)^{\prime}+\gamma_{T-1} E\left(Q_{T-2} R_{T-2}^{\prime}\right)\right] l_{T-2}\right. \\
& \left.\quad+\frac{\lambda_{T-2}^{1}}{2} \mu_{T-2}^{\prime}\right\} u_{T-2} \\
& +\left[\operatorname{Var}\left(Q_{T-2}\right)+\beta_{2, T-1} E\left(Q_{T-2}^{2}\right)\right] l_{T-2}^{2} \\
& +\left[\lambda_{T-2} E\left(Q_{T-2}\right)+\beta_{1, T-1} E\left(Q_{T-2}\right)\right] l_{T-1} \\
& -\frac{\left(\lambda_{T-1}\right)^{2}}{4} \alpha_{T-1} .
\end{aligned}
$$

By setting $\omega_{T-1}$ be the Lagrange multiplier, the Lagrange function for problem (23) is

$$
\begin{aligned}
L\left(u_{T-2}\right):= & u_{T-2}^{\prime} \Sigma_{T-2}^{1} u_{T-2}-2 \Phi_{T-2}^{\prime} u_{T-2} \\
& +\Psi_{T-2}-\omega_{T-1}\left(x_{T-2}-I^{\prime} u_{T-2}\right)
\end{aligned}
$$

where

$$
\begin{aligned}
& \Phi_{T-2}:=\left[\Sigma_{T-2}^{0}+\gamma_{T-1} E\left(Q_{T-2} R_{T-2}\right)\right] l_{T-2}+\frac{\lambda_{T-2}^{1}}{2} \mu_{T-2} \\
& \Psi_{T-2}:= {\left[\operatorname{Var}\left(Q_{T-2}\right)+\beta_{2, T-1} E\left(Q_{T-2}^{2}\right)\right] l_{T-2}^{2} } \\
&+\left[\lambda_{T-2} E\left(Q_{T-2}\right)+\beta_{1, T-1} E\left(Q_{T-2}\right)\right] l_{T-1} \\
&-\frac{\left(\lambda_{T-1}\right)^{2}}{4} \alpha_{T-1} .
\end{aligned}
$$

From the first-order necessary optimality condition, we have

$$
\begin{gathered}
2 \Sigma_{T-2}^{1} u_{T-2}-2 \Phi_{T-2}+\omega_{T-1} I=0, \\
x_{T-2}-I^{\prime} u_{T-2}=0 .
\end{gathered}
$$

From (27), we have

$$
\begin{aligned}
u_{T-2}^{*} & =\left(\Sigma_{T-2}^{1}\right)^{-1} \Phi_{T-2}-\frac{1}{2} \omega_{T-1}\left(\Sigma_{T-2}^{1}\right)^{-1} I, \\
I^{\prime} u_{T-2}^{*}= & I^{\prime}\left(\Sigma_{T-2}^{1}\right)^{-1} \Phi_{T-2}-\frac{1}{2} \omega_{T-1} I^{\prime}\left(\Sigma_{T-2}^{1}\right)^{-1} I \\
& =I^{\prime}\left(\Sigma_{T-2}^{1}\right)^{-1} \Phi_{T-2}-\frac{1}{2} \omega_{T-1} a_{T-2} \\
& =x_{T-2},
\end{aligned}
$$

which implies

$$
\omega_{T-1}=2 \frac{I^{\prime}\left(\Sigma_{T-2}^{1}\right)^{-1} \Phi_{T-2}}{a_{T-2}}-2 \frac{1}{a_{T-2}} x_{T-2} .
$$

Substituting $\omega_{T-1}$ into (29), we get

$$
\begin{aligned}
u_{T-2}^{*}= & \frac{\left(\Sigma_{T-2}^{1}\right)^{-1} I}{a_{T-2}} x_{T-2}+\left(\Sigma_{T-2}^{1}\right)^{-1} \Phi_{T-2} \\
& -\frac{I^{\prime}\left(\Sigma_{T-2}^{1}\right)^{-1} \Phi_{T-2}}{a_{T-2}}\left(\Sigma_{T-2}^{1}\right)^{-1} I .
\end{aligned}
$$

Taking $\Phi_{T-2}$ into account, we have

$$
\begin{aligned}
u_{T-2}^{*}= & \frac{\left(\Sigma_{T-2}^{1}\right)^{-1} I}{a_{T-2}} x_{T-2}+\frac{\lambda_{T-2}^{1}}{2}\left(\Sigma_{T-2}^{1}\right)^{-1}\left(\mu_{T-2}-q_{T-2} I\right) \\
& +\left(\Sigma_{T-2}^{1}\right)^{-1}\left[\Sigma_{T-2}^{0}+\gamma_{T-1} E\left(Q_{T-2} R_{T-2}\right)-\widetilde{\gamma}_{T-2} I\right] l_{T-2} .
\end{aligned}
$$


Substituting $u_{T-2}^{*}$ into (23) gives (see Appendix B for more details)

$$
\begin{aligned}
V_{T-2}^{*}\left(x_{T-2}, l_{T-2}\right)= & \frac{1}{a_{T-2}} x_{T-2}^{2}-\lambda_{T-2}^{1} q_{T-2} x_{T-2} \\
& -2 \tilde{\gamma}_{T-2} x_{T-2} l_{T-2}+\beta_{1, T-2} l_{T-2}+\beta_{2, T-2} l_{T-2}^{2} \\
& -\frac{\left(\lambda_{T-1}\right)^{2}}{4} \alpha_{T-1}-\frac{\left(\lambda_{T-2}^{1}\right)^{2}}{4} \alpha_{T-2} .
\end{aligned}
$$

Next, by using mathematical induction, we show that both (10) and

$$
\begin{aligned}
V_{t}^{*}\left(x_{t}, l_{t}\right)= & \frac{1}{a_{t}} x_{t}^{2}-\lambda_{t}^{1} q_{t} x_{t}-2 \widetilde{\gamma}_{t} x_{t} l_{t} \\
& +\beta_{1, t} l_{t}+\beta_{2, t} l_{t}^{2}-\sum_{i=t}^{T-1} \frac{\left(\lambda_{i}^{1}\right)^{2}}{4} \alpha_{i}
\end{aligned}
$$

hold. Suppose that (10) and (35) hold for time $t, t+1, \ldots, T-1$. At the beginning of $(t-1)$ th period, for given wealth $x_{t-1}$ and liability $l_{t-1}$, the corresponding optimal investment problem is

$$
\begin{array}{ll}
\min & \operatorname{Var}_{t-1}\left(S_{t}\right)-\lambda_{t-1} E_{t-1}\left(S_{t}\right) \\
& +E_{t-1}\left(V_{t}^{*}\left(x_{t}, l_{t}\right)\right) \\
\text { s.t. } & R_{t-1}^{\prime} u_{t-1}=x_{t}, \\
& I^{\prime} u_{t-1}=x_{t-1}, \\
& l_{t}=Q_{t-1} l_{t-1}, \\
& S_{t}=x_{t}-l_{t} .
\end{array}
$$

It follows from (35) that

$$
\begin{aligned}
E_{t-1}\left(V_{t}^{*}\left(x_{t}, l_{t}\right)\right)= & \frac{1}{a_{t}} E_{t-1}\left(x_{t}^{2}\right)-\lambda_{t}^{1} q_{t} E_{t-1}\left(x_{t}\right) \\
& -2 \widetilde{\gamma}_{t} E_{t-1}\left(x_{t} l_{t}\right)+\beta_{1, t} E_{t-1}\left(l_{t}\right) \\
& +\beta_{2, t} E_{t-1}\left(l_{t}^{2}\right)-\sum_{i=t}^{T-1} \frac{\left(\lambda_{i}^{1}\right)^{2}}{4} \alpha_{i} .
\end{aligned}
$$

Substituting $E_{t-1}\left(V_{t}^{*}\left(x_{t}, l_{t}\right)\right)$ and the binding constraints into the objective function of problem (36), we have

$$
\begin{array}{ll}
\min & V_{t-1}\left(x_{t-1}, l_{t-1}\right):=u_{T-1}^{\prime} \Sigma_{t-1}^{1} u_{t-1}-2 \Phi_{t-1}^{\prime} u_{t-1}+\Psi_{t-1} \\
\text { s.t. } & I^{\prime} u_{t-1}=x_{t-1},
\end{array}
$$

where

$$
\begin{aligned}
\Phi_{t-1}: & =\left[\Sigma_{t-1}^{0}+\widetilde{\gamma}_{t} E\left(Q_{t-1} R_{t-1}\right)\right] l_{t-1}+\frac{\lambda_{t-1}^{1}}{2} \mu_{t-1}, \\
\Psi_{t-1}:= & {\left[\operatorname{Var}\left(Q_{t-1}\right)+\beta_{2, t} E\left(Q_{t-1}^{2}\right)\right] l_{t-1}^{2} } \\
& +\left[\lambda_{t-1}+\beta_{1, t}\right] E\left(Q_{t-1}\right) l_{t-1}-\sum_{i=t}^{T-1} \frac{\left(\lambda_{i}^{1}\right)^{2}}{4} \alpha_{i} .
\end{aligned}
$$

Letting $\omega_{t}$ be the Lagrange multiplier, the Lagrange function for problem (38) is given by

$$
\begin{aligned}
L\left(u_{t-1}\right):= & u_{T-1}^{\prime} \Sigma_{t-1}^{1} u_{t-1}-2 \Phi_{t-1}^{\prime} u_{t-1} \\
& +\Psi_{t-1}-\omega_{t}\left(x_{t-1}-I^{\prime} u_{t-1}\right) .
\end{aligned}
$$

It follows from the first-order necessary optimality condition that

$$
\begin{gathered}
2 \Sigma_{t-1}^{1} u_{t-1}-2 \Phi_{t-1}+\omega_{t} I=0 \\
x_{t-1}-I^{\prime} u_{t-1}=0
\end{gathered}
$$

Thus, we have

$$
\begin{aligned}
u_{t-1}^{*} & =\left(\Sigma_{t-1}^{1}\right)^{-1} \Phi_{t-1}-\frac{1}{2} \omega_{t}\left(\Sigma_{t-1}^{1}\right)^{-1} I, \\
I^{\prime} u_{t-1}^{*} & =I^{\prime}\left(\Sigma_{t-1}^{1}\right)^{-1} \Phi_{t-1}-\frac{1}{2} \omega_{t} I^{\prime}\left(\Sigma_{t-1}^{1}\right)^{-1} I \\
& =I^{\prime}\left(\Sigma_{t-1}^{1}\right)^{-1} \Phi_{t-1}-\frac{1}{2} \omega_{t} a_{t-1} \\
& =x_{t-1},
\end{aligned}
$$

which implies

$$
\omega_{t}=2 \frac{I^{\prime}\left(\Sigma_{t-1}^{1}\right)^{-1} \Phi_{t-1}}{a_{t-1}}-2 \frac{1}{a_{t-1}} x_{t-1} .
$$

It follows from (42) that

$$
\begin{aligned}
u_{t-1}^{*}= & \frac{\left(\Sigma_{t-1}^{1}\right)^{-1} I}{a_{t-1}} x_{t-1}-\frac{I^{\prime}\left(\Sigma_{t-1}^{1}\right)^{-1} \Phi_{t-1}}{a_{t-1}}\left(\Sigma_{t-1}^{1}\right)^{-1} I \\
& +\left(\Sigma_{t-1}^{1}\right)^{-1} \Phi_{t-1} \\
= & \frac{\left(\Sigma_{t-1}^{1}\right)^{-1} I}{a_{t-1}} x_{t-1}+\frac{\lambda_{t-1}^{1}}{2}\left(\Sigma_{t-1}^{1}\right)^{-1}\left(\mu_{t-1}-q_{t-1} I\right) \\
& +\left(\Sigma_{t-1}^{1}\right)^{-1}\left[\Sigma_{t-1}^{0}-\widetilde{\gamma}_{t-1} I+\widetilde{\gamma_{t}} E\left(Q_{t-1} R_{t-1}\right)\right] l_{t-1} .
\end{aligned}
$$

Substituting $u_{t-1}^{*}$ into the objective function of problem (38) (see Appendix C for more details), we have

$$
\begin{aligned}
V_{t-1}^{*}\left(x_{t-1}, l_{t-1}\right)= & \frac{1}{a_{t-1}} x_{t-1}^{2}-\lambda_{t-1}^{1} q_{t-1} x_{t-1}-2 \tilde{\gamma}_{t-1} x_{t-1} l_{t-1} \\
& +\beta_{1, t-1} l_{t-1}+\beta_{2, t-1} l_{t-1}^{2}-\sum_{i=t-1}^{T-1} \frac{\left(\lambda_{i}^{1}\right)^{2}}{4} \alpha_{i} .
\end{aligned}
$$

This completes the proof. 
Remark 2. If an investor does not have any liability, that is, $l_{t} \equiv 0$ for any $t \in\{0,1, \ldots, T-1\}$, then the optimal timeconsistent investment strategy can be simplified as follows:

$$
u_{t}^{*}=\frac{\left(\Sigma_{t}^{1}\right)^{-1} I}{a_{t}} x_{t}+\frac{\lambda_{t}^{1}}{2}\left(\Sigma_{t}^{1}\right)^{-1}\left(\mu_{t}-q_{t} I\right), \quad t=0, \ldots, T-1 \text {, }
$$

which is exactly the same as that in [16]. This implies that the result of Chen et al. [16] is a special case of Theorem 1. Therefore, Theorem 1 generalizes their result.

Corollary 3. If the returns of liability and risky assets are uncorrelated, that is, $\Sigma_{t}^{0}=0$ for any $t \in\{0,1, \ldots, T-1\}$, then the optimal investment policy for problem (9) is

$$
u_{t}^{*}=\frac{\left(\Sigma_{t}^{1}\right)^{-1} I}{a_{t}} x_{t}+\frac{\lambda_{t}^{1}}{2}\left(\Sigma_{t}^{1}\right)^{-1}\left(\mu_{t}-q_{t} I\right), \quad t=0, \ldots, T-1 .
$$

Proof. Since $\Sigma_{t}^{0}=0$, it is easy to verify that $d_{t}=I^{\prime}\left(\Sigma_{t}^{1}\right)^{-1} \Sigma_{t}^{0}=$ $0, \gamma_{t}=d_{t} / a_{t}=0$ and $\tilde{\gamma}_{t}=0$. Substituting them into (10) gives

$$
u_{t}^{*}=\frac{\left(\Sigma_{t}^{1}\right)^{-1} I}{a_{t}} x_{t}+\frac{\lambda_{t}^{1}}{2}\left(\Sigma_{t}^{1}\right)^{-1}\left(\mu_{t}-q_{t} I\right), \quad t=0, \ldots, T-1 .
$$

This completes the proof.

Remark 4. After comparing Corollary 3 and Remark 2, it is quite clear that, if the return of liability is uncorrelated with that of risky asset, then the liability does not affect the timeconsistent optimal policy in a market without riskless asset.

Remark 5. If the return of liability is correlated to those of risky assets, then the occurrence of liability leads to a parallel shift of the optimal investment policy and the shift is

$$
\left(\Sigma_{t}^{1}\right)^{-1}\left(\Sigma_{t}^{0}-\widetilde{\gamma}_{t} I+\widetilde{\gamma}_{t+1} E\left(Q_{t} R_{t}\right)\right) l_{t}
$$

which depends on the current value of liability, $l_{t}$, and the covariance between the returns of liability and risky assets, $\Sigma_{t}^{0}$.

Next, we compare the time-consistent strategy with the myopic strategy in a market without riskless asset. In such a market, problem (2) can be expressed as follows:

$$
\begin{array}{ll}
\min _{u_{t}} & \operatorname{Var}_{t}\left(S_{t+1}\right)-\lambda_{t} E_{t}\left(S_{t+1}\right) \\
\text { s.t. } & R_{t}^{\prime} u_{t}=x_{t+1}, \\
& I^{\prime} u_{t}=x_{t}, \\
& l_{t+1}=Q_{t} l_{t}, \\
& S_{t+1}=x_{t+1}-l_{t+1} .
\end{array}
$$

By using the same method in the proof of Theorem 1 for time $T-1$, the myopic strategy is given by

$$
\begin{aligned}
u_{t}^{m y}:= & \frac{\left(\Sigma_{t}\right)^{-1} I}{\bar{a}_{t}} x_{t}+\frac{\lambda_{t}}{2}\left(\Sigma_{t}\right)^{-1}\left(\mu_{t}-\bar{q}_{t} I\right) \\
& +\left(\Sigma_{t}\right)^{-1}\left(\Sigma_{t}^{0}-\bar{\gamma}_{t} I\right) l_{t}, \quad t=0, \ldots, T-1,
\end{aligned}
$$

where $\bar{a}_{t}=I^{\prime}\left(\Sigma_{t}\right)^{-1} I, \bar{q}_{t}=I^{\prime}\left(\Sigma_{t}\right)^{-1} \mu_{t} / \bar{a}_{t}, \bar{\gamma}_{t}=I^{\prime}\left(\Sigma_{t}\right)^{-1} \Sigma_{t}^{0} / \bar{a}_{t}$. It is clear that the difference between two strategies enters into all of the three parts. More specifically, the following feature holds; if the investor is arbitrarily risk averse, that is, $\lambda_{t} \rightarrow 0$, then both the time consistent optimal strategy and myopic strategy reduce to

$$
\begin{gathered}
u_{t}^{*}=\frac{\left(\Sigma_{t}^{1}\right)^{-1} I}{a_{t}} x_{t}+\left(\Sigma_{t}^{1}\right)^{-1}\left(\Sigma_{t}^{0}-\widetilde{\gamma}_{t} I+\widetilde{\gamma}_{t+1} E\left(Q_{t} R_{t}\right)\right) l_{t}, \\
t=0, \ldots, T-1, \\
u_{t}^{m y}=\frac{\left(\Sigma_{t}\right)^{-1} I}{\bar{a}_{t}} x_{t}+\left(\Sigma_{t}\right)^{-1}\left(\Sigma_{t}^{0}-\bar{\gamma}_{t} I\right) l_{t}, \quad t=0, \ldots, T-1,
\end{gathered}
$$

respectively. After comparing these two strategies, we find that, if an investor is arbitrarily risk averse, then he/she should concern about the time-diversification effects arising from multiperiod optimization.

\section{Time Consistent Optimal Strategy with Riskless Asset}

In this section, we consider a market which is consisting of one riskless asset and $n$ risky assets and assume that the wealth process $x_{t}$ is also in a self-financing fashion. We list the notations of this section in Table 2 . The wealth process $x_{t}$ can be described as follows:

$$
x_{t}=P_{t-1}^{\prime} u_{t-1}+s_{t-1} x_{t-1},
$$

where $P_{t}=R_{t}-s_{t} I$. In this setting, problem (6) can be written as follows:

$$
\begin{array}{ll}
\min & \rho_{0}\left(S_{1}\right)+E_{0}\left(\rho_{1}\left(S_{2}\right)+E_{1}\left(\rho_{2}\left(S_{3}\right)+\cdots\right.\right. \\
& \left.\left.+E_{T-3}\left(\rho_{T-2}\left(S_{T-1}\right)+E_{T-2}\left(\rho_{T-1}\left(S_{T}\right)\right)\right) \ldots\right)\right) \\
\text { s.t. } & x_{t+1}=P_{t}^{\prime} u_{t}+s_{t} x_{t}, \\
& l_{t+1}=Q_{t} l_{t}, \\
& S_{t+1}=x_{t+1}-l_{t+1}, \quad t=0,1, \ldots, T-1 .
\end{array}
$$

By applying Bellman's optimality principle, the timeconsistent optimal investment policy of problem (55) is given in the following theorem.

Theorem 6. The optimal investment strategy of problem (55) is given by

$$
u_{t}^{*}=\Sigma_{t}^{-1} \varphi_{t} l_{t}+\frac{\lambda_{t}^{1}}{2} \Sigma_{t}^{-1} \epsilon_{t}, \quad t=0, \ldots, T-1,
$$

where $\varphi_{t}=E\left(P_{t} Q_{t}\right)-E\left(P_{t}\right) E\left(Q_{t}\right)$ and $\epsilon_{t}=E\left(P_{t}\right)$. 
TABLE 2: Model notations of Section 4.

\begin{tabular}{ll}
\hline For $t=T-1:$ & For $t=T-2, \ldots, 0:$ \\
$\lambda_{T-1}^{1}=\lambda_{T-1}$ & $\lambda_{t}^{1}=\lambda_{t}+\lambda_{t+1}^{1} s_{t+1}$ \\
$\bar{\beta}_{1, T-1}=\lambda_{T-1}\left[E\left(Q_{T-1}\right)-\epsilon_{T-1}^{\prime}\left(\Sigma_{T-1}\right)^{-1} \varphi_{T-1}\right]$ & $\bar{\beta}_{1, t}=\left(\lambda_{t}+\beta_{1, t+1}\right) E\left(Q_{t}\right)-\lambda_{t}^{1} \epsilon_{t}^{\prime}\left(\Sigma_{t}\right)^{-1} \varphi_{t}$ \\
$\bar{\beta}_{2, T-1}=\operatorname{Var}\left(Q_{T-1}\right)-\varphi_{T-1}^{\prime}\left(\Sigma_{T-1}\right)^{-1} \varphi_{T-1}$ & $\bar{\beta}_{2, t}=\operatorname{Var}\left(Q_{t}\right)+\beta_{2, t+1} E\left(Q_{t}^{2}\right)-\varphi_{t}^{\prime}\left(\Sigma_{t}\right)^{-1} \varphi_{t}$ \\
\hline
\end{tabular}

Proof. When $t=T-1$, for given wealth $x_{T-1}$ and liability $l_{T-1}$ at the beginning of the $(T-1)$ th period, problem (55) reduces to

$$
\begin{array}{ll}
\min _{u_{T-1}} & \operatorname{Var}_{T-1}\left(S_{T}\right)-\lambda_{T-1} E_{T-1}\left(S_{T}\right) \\
\text { s.t. } & x_{T}=s_{T-1} x_{T-1}+P_{T-1}^{\prime} u_{T-1}, \\
& l_{T}=Q_{T-1} l_{T-1}, \\
& S_{T}=x_{T}-l_{T} .
\end{array}
$$

Substituting the binding constraints into the objective function, we have

$$
\min _{u_{T-1}} V_{T-1}\left(x_{T-1}, l_{T-1}\right)
$$

where

$$
\begin{aligned}
V_{T-1}\left(x_{T-1}, l_{T-1}\right):= & u_{T-1}^{\prime} \Sigma_{T-1} u_{T-1} \\
& -2\left(\varphi_{T-1}^{\prime} l_{T-1}+\frac{\lambda_{T-1}}{2} \epsilon_{T-1}^{\prime}\right) u_{T-1} \\
& +\operatorname{Var}\left(Q_{T-1}\right) l_{T-1}^{2}-\lambda_{T-1} s_{T-1} x_{T-1} \\
& +\lambda_{T-1} E\left(Q_{T-1}\right) l_{T-1} .
\end{aligned}
$$

It is clear that problem (58) is an unconstrained convex program problem. By using the first-order necessary optimality condition, we have

$$
\begin{aligned}
\frac{d\left(V_{T-1}\left(x_{T-1}, l_{T-1}\right)\right)}{d\left(u_{T-1}\right)}= & 2 \Sigma_{T-1} u_{T-1} \\
& -2\left(\varphi_{T-1} l_{T-1}+\frac{\lambda_{T-1}}{2} \epsilon_{T-1}\right) \\
= & 0,
\end{aligned}
$$

which implies

$$
u_{T-1}^{*}=\left(\Sigma_{T-1}\right)^{-1}\left[\varphi_{T-1} l_{T-1}+\frac{\lambda_{T-1}}{2} \epsilon_{T-1}\right] .
$$

Substituting $u_{T-1}^{*}$ into the objective function of problem (58) gives (see Appendix D for more details)

$$
\begin{aligned}
V_{T-1}^{*}\left(x_{T-1}, l_{T-1}\right)= & \bar{\beta}_{2, T-1} l_{T-1}^{2}+\bar{\beta}_{1, T-1} l_{T-1} \\
& -\lambda_{T-1} s_{T-1} x_{T-1}-\frac{\left(\lambda_{T-1}\right)^{2}}{4} M_{T-1},
\end{aligned}
$$

where $M_{T-1}=\epsilon_{T-1}^{\prime} \Sigma_{T-1}^{-1} \epsilon_{T-1}$.
When $t=T-2$, for given wealth $x_{T-2}$ and liability $l_{T-2}$ at the beginning of the $(T-2)$ th period, the corresponding optimal investment problem can be expressed as follows:

$$
\begin{aligned}
& \min \quad \operatorname{Var}_{T-2}\left(S_{T-1}\right)-\lambda_{T-2} E_{T-2}\left(S_{T-1}\right) \\
&+E_{T-2}\left(V_{T-1}^{*}\left(x_{T-1}, l_{T-1}\right)\right) \\
& \text { s.t. } \quad x_{T-1}=s_{T-2} x_{T-2}+P_{T-2}^{\prime} u_{T-2}, \\
& l_{T-1}=Q_{T-2} l_{T-2}, \\
& S_{T-1}=x_{T-1}-l_{T-1} .
\end{aligned}
$$

From (62), we can easily have

$$
\begin{aligned}
E_{T-2}\left(V_{T-1}^{*}\left(x_{T-1}, l_{T-1}\right)\right)= & \bar{\beta}_{2, T-1} E\left(Q_{T-2}^{2}\right) l_{T-2}^{2} \\
& +\bar{\beta}_{1, T-1} E\left(Q_{T-2}\right) l_{T-2} \\
& -\lambda_{T-1} s_{T-1} s_{T-2} x_{T-2} \\
& -\lambda_{T-1} s_{T-1} \epsilon_{T-2}^{\prime} u_{T-2} \\
& -\frac{\left(\lambda_{T-1}\right)^{2}}{4} M_{T-1} .
\end{aligned}
$$

Substituting $E_{T-2}\left(V_{T-1}^{*}\left(x_{T-1}, l_{T-1}\right)\right)$ and the binding constraints into the objective function of problem (63), we have

$$
\min _{u_{T-2}} V_{T-2}\left(x_{T-2}, l_{T-2}\right),
$$

where

$$
\begin{aligned}
V_{T-2}\left(x_{T-2}, l_{T-2}\right)= & u_{T-2}^{\prime} \Sigma_{T-2} u_{T-2} \\
& -2\left(\varphi_{T-2}^{\prime} l_{T-2}+\frac{\lambda_{T-2}^{1}}{2} \epsilon_{T-2}^{\prime}\right) u_{T-2} \\
& +\left[\operatorname{Var}\left(Q_{T-2}\right)+\bar{\beta}_{2, T-1} E\left(Q_{T-2}^{2}\right)\right] l_{T-2}^{2} \\
& +\left[\lambda_{T-2} E\left(Q_{T-2}\right)+\bar{\beta}_{1, T-1} E\left(Q_{T-2}\right)\right] l_{T-1} \\
& -\lambda_{T-2}^{1} s_{T-2} x_{T-2}-\frac{\left(\lambda_{T-1}\right)^{2}}{4} M_{T-1} .
\end{aligned}
$$

The first-order necessary optimality condition implies

$$
\begin{aligned}
\frac{d\left(V_{T-2}\left(x_{T-2}, l_{T-2}\right)\right)}{d\left(u_{T-2}\right)}= & 2 \Sigma_{T-2} u_{T-2} \\
& -2\left(\varphi_{T-2} l_{T-2}+\frac{\lambda_{T-2}^{1}}{2} \epsilon_{T-2}\right)
\end{aligned}
$$


Thus,

$$
\begin{aligned}
u_{T-2}^{*} & =\left(\Sigma_{T-2}\right)^{-1}\left(\varphi_{T-2} l_{T-2}+\frac{\lambda_{T-2}^{1}}{2} \epsilon_{T-2}\right) \\
& =\left(\Sigma_{T-2}\right)^{-1} \varphi_{T-2} l_{T-2}+\frac{\lambda_{T-2}^{1}}{2}\left(\Sigma_{T-2}\right)^{-1} \epsilon_{T-2} .
\end{aligned}
$$

Substituting $u_{T-2}^{*}$ into the objective function of problem (65) (see Appendix E for more details), we have

$$
\begin{aligned}
V_{T-2}^{*}\left(x_{T-2}, l_{T-2}\right)= & \bar{\beta}_{2, T-2} l_{T-2}^{2}+\bar{\beta}_{1, T-2} l_{T-2}-\lambda_{T-2}^{1} s_{T-2} x_{T-2} \\
& -\frac{\left(\lambda_{T-1}\right)^{2}}{4} M_{T-1}-\frac{\left(\lambda_{T-2}^{1}\right)^{2}}{4} M_{T-2},
\end{aligned}
$$

where $M_{T-2}=\epsilon_{T-2}^{\prime} \Sigma_{T-2}^{-1} \epsilon_{T-2}$.

Next, by using mathematical induction, we show that both (56) and

$$
V_{t}^{*}\left(x_{t}, l_{t}\right)=\bar{\beta}_{2, t} l_{t}^{2}+\bar{\beta}_{1, t} l_{t}-\lambda_{t}^{1} s_{t} x_{t}-\sum_{i=t}^{T-1} \frac{\left(\lambda_{i}^{1}\right)^{2}}{4} M_{i}
$$

hold, where $M_{i}=\epsilon_{i}^{\prime} \Sigma_{i}^{-1} \epsilon_{i}$ with $i=0,1, \ldots, T-1$. Suppose that (56) and (70) are true for time $t, t+1, \ldots, T-1$. At the beginning of the $(t-1)$ th period, for given wealth $x_{t-1}$ and liability $l_{t-1}$, the corresponding optimal investment problem is

$$
\begin{array}{ll}
\min & \operatorname{Var}_{t-1}\left(S_{t}\right)-\lambda_{t-1} E_{t-1}\left(S_{t}\right)+E_{t-1}\left(V_{t}^{*}\left(x_{t}, l_{t}\right)\right) \\
\text { s.t. } & x_{t}=s_{t-1} x_{t-1}+P_{t-1}^{\prime} u_{t-1}, \\
& l_{t}=Q_{t-1} l_{t-1} \\
& S_{t}=x_{t}-l_{t} .
\end{array}
$$

It follows from (70) that

$$
\begin{aligned}
E_{t-1}\left(V_{t}^{*}\left(x_{t}, l_{t}\right)\right)= & \bar{\beta}_{2, t} E\left(Q_{t-1}^{2}\right) l_{t-1}^{2}+\bar{\beta}_{1, t} E\left(Q_{t-1}\right) l_{t} \\
& -\lambda_{t}^{1} s_{t} s_{t-1} x_{t-1}-\lambda_{t}^{1} s_{t} \epsilon_{t-1}^{\prime} u_{t-1} \\
& -\sum_{i=t}^{T-1} \frac{\left(\lambda_{i}^{1}\right)^{2}}{4} M_{i} .
\end{aligned}
$$

Substituting $E_{t-1}\left(V_{t}^{*}\left(x_{t}, l_{t}\right)\right)$ and the binding constraints into the objective function of problem (71), we have

$$
\min _{u_{t-1}} V_{t-1}\left(x_{t-1}, l_{t-1}\right) \text {, }
$$

where

$$
\begin{aligned}
V_{t-1}\left(x_{t-1}, l_{t-1}\right)= & u_{t-1}^{\prime} \Sigma_{t-1} u_{t-1}-2\left(\varphi_{t-1}^{\prime} l_{t-1}+\frac{\lambda_{t-1}^{1}}{2} \epsilon_{t-1}^{\prime}\right) u_{t-1} \\
& +\left[\operatorname{Var}\left(Q_{t-1}\right)+\bar{\beta}_{2, t} E\left(Q_{t-1}^{2}\right)\right] l_{t-1}^{2} \\
& +\left[\lambda_{t-1} E\left(Q_{t-1}\right)+\bar{\beta}_{1, t} E\left(Q_{t-1}\right)\right] l_{t-1} \\
& -\lambda_{t-1}^{1} s_{t-1} x_{t-1}-\sum_{i=t}^{T-1} \frac{\left(\lambda_{i}^{1}\right)^{2}}{4} M_{i} .
\end{aligned}
$$

The first-order necessary optimality condition gives

$$
\begin{aligned}
\frac{d\left(V_{t-1}\left(x_{t-1}, l_{t-1}\right)\right)}{d\left(u_{t-1}\right)} & =2 \Sigma_{t-1} u_{t-1}-2\left(\varphi_{t-1} l_{t-1}+\frac{\lambda_{t-1}^{1}}{2} \epsilon_{t-1}\right) \\
& =0
\end{aligned}
$$

which implies

$$
u_{t-1}^{*}=\Sigma_{t-1}^{-1} \varphi_{t-1} l_{t-1}+\frac{\lambda_{t-1}^{1}}{2} \Sigma_{t-1}^{-1} \epsilon_{t-1} .
$$

Substituting $u_{t-1}^{*}$ into the objective function of problem (73) (see Appendix F for more details), we have

$$
\begin{aligned}
V_{t-1}^{*}\left(x_{t-1}, l_{t-1}\right)= & \bar{\beta}_{2, t-1} l_{t-1}^{2}+\bar{\beta}_{1, t-1} l_{t-1} \\
& -\lambda_{t-1}^{1} s_{t-1} x_{t-1}-\sum_{i=t-1}^{T-1} \frac{\left(\lambda_{i}^{1}\right)^{2}}{4} M_{i} .
\end{aligned}
$$

This completes the proof.

Remark 7. From Theorem 6, it is clear that, if there is a riskless asset in the market, then the time-consistent optimal investment policy is wholly independent of the current wealth $x_{t}$. However, Theorem 1 gives an opposite conclusion. This implies that the riskless asset does affect the optimal strategy. Therefore, an investor should carefully select the market they invested.

Remark 8. If there is no liability, that is, $l_{t} \equiv 0$ for any $t \in$ $\{0,1, \ldots, T-1\}$, then the time-consistent optimal investment policy reduces to

$$
u_{t}^{*}=\frac{\lambda_{t}^{1}}{2} \Sigma_{t}^{-1} \epsilon_{t}, \quad t=0, \ldots, T-1,
$$

which is the same as that in [16]. This implies that the result of Chen et al. [16] is a special case of Theorem 6. Therefore, Theorem 6 generalizes their result.

Corollary 9. If the return of liability, $Q_{t}$, is uncorrelated with those of risky assets, $R_{t}$, that is, $\Sigma_{t}^{0}=0$ for any $t \in\{0,1, \ldots, T-$ $1\}$, then the optimal policy for problem (55) is

$$
u_{t}^{*}=\frac{\lambda_{t}^{1}}{2} \Sigma_{t}^{-1} \epsilon_{t}, \quad t=0, \ldots, T-1 .
$$

Proof. Since $\Sigma_{t}^{0}=0$, it is easy to have

$$
\begin{aligned}
\varphi_{t} & =E\left(P_{t} Q_{t}\right)-E\left(P_{t}\right) E\left(Q_{t}\right) \\
& =E\left(\left(R_{t}-s_{t} I\right) Q_{t}\right)-E\left(R_{t}-s_{t} I\right) E\left(Q_{t}\right) \\
& =E\left(R_{t} Q_{t}\right)-E\left(R_{t}\right) E\left(Q_{t}\right) \\
& =\Sigma_{t}^{0} \\
& =0 .
\end{aligned}
$$


Substituting $\varphi_{t}=0$ into (56) gives

$$
u_{t}^{*}=\frac{\lambda_{t}^{1}}{2} \Sigma_{t}^{-1} \epsilon_{t}, \quad t=0, \ldots, T-1 .
$$

This completes the proof.

Remark 10. After comparing Corollary 9 and Remark 8, it is quite clear that, if the return of liability is uncorrelated with those of risky assets, then the occurrence of liability does not affect the time-consistent optimal investment policy in a market with riskless asset.

Remark 11. If the return of liability is correlated with those of risky assets, then the occurrence of liability leads to a parallel shift of the optimal investment policy and the shift is

$$
\Sigma_{t}^{-1} \varphi_{t} l_{t}
$$

which depends on the current value of the liability.

Now, we compare the time-consistent policy with the myopic strategy in a market with a riskless asset. In such a market, problem (2) can be further expressed as follows:

$$
\begin{array}{ll}
\min _{u_{t}} & \operatorname{Var}_{t}\left(S_{t+1}\right)-\lambda_{t} E_{t}\left(S_{t+1}\right) \\
\text { s.t. } & x_{t+1}=s_{t} x_{t}+P_{t}^{\prime} u_{t} \\
& l_{t+1}=Q_{t} l_{t} \\
& S_{t+1}=x_{t+1}-l_{t+1} .
\end{array}
$$

By using the same method in the proof of Theorem 6 for time $T-1$, the myopic strategy is given by

$$
u_{t}^{m y}:=\Sigma_{t}^{-1} \varphi_{t} l_{t}+\frac{\lambda_{t}}{2} \Sigma_{t}^{-1} \epsilon_{t}, \quad t=0, \ldots, T-1 .
$$

Different with the market without riskless asset, the difference between the time-consistent optimal strategy and the myopic strategy only enters in the part depending on the risk aversion $\lambda_{t}$. Further, the following feature holds; if an investor is arbitrarily risk averse, that is, $\lambda_{t} \rightarrow 0$, then both the timeconsistent optimal investment policy and the myopic strategy reduce to

$$
u_{t}^{*}=\Sigma_{t}^{-1} \varphi_{t} l_{t}, \quad t=0, \ldots, T-1
$$

This implies that, if an investor is arbitrarily risk averse, then he/she could ignore the time-diversification effects arising from multiperiod optimization. Further, if the investor does not have any liability, then both two strategies suggest that he/she should leave the market.

Remark 12. After comparing the results of these two different markets, we find that, for an arbitrarily risk averse investor, if there is a riskless asset in the market, the time-diversification effects could be ignored, otherwise, the effects should be considered.
TABLE 3: Time-consistent strategies with and without liability for $\lambda_{0}=\lambda_{1}=0.5$.

\begin{tabular}{ccc}
\hline$t$ & $\begin{array}{c}\text { Time-consistent strategy with } \\
\text { liability } u_{t}^{*}\end{array}$ & $\begin{array}{c}\text { Time-consistent strategy } \\
\text { without liability } \tilde{u}_{t}^{*}\end{array}$ \\
\hline 0 & $\left(\begin{array}{c}1.194 l_{0}+0.903 x_{0}+3.736 \\
0.107 l_{0}-0.066 x_{0}+0.203 \\
-0.577 l_{0}+0.163 x_{0}-3.939\end{array}\right)$ & $\left(\begin{array}{c}0.903 x_{0}+3.736 \\
-0.066 x_{0}+0.203 \\
0.163 x_{0}-3.939\end{array}\right)$ \\
\hline 1 & $\left(\begin{array}{c}-0.372 l_{1}+1.018 x_{1}+1.743 \\
0.198 l_{1}-0.060 x_{1}+0.095 \\
-0.633 l_{1}+0.042 x_{1}-1.838\end{array}\right)$ & $\left(\begin{array}{c}1.018 x_{1}+1.743 \\
-0.060 x_{1}+0.095 \\
0.042 x_{1}-1.838\end{array}\right)$ \\
\hline
\end{tabular}

TABLE 4: Investment strategies in a market without riskless asset for $\lambda_{0}=\lambda_{1}=0.5$.

\begin{tabular}{lcc}
\hline$t$ & Time-consistent strategy $u_{t}^{*}$ & Myopic strategy $u_{t}^{m y}$ \\
$0\left(\begin{array}{c}0.903 x_{0}+1.194 l_{0}+3.736 \\
-0.066 x_{0}+0.107 l_{0}+0.203 \\
0.163 x_{0}-0.577 l_{0}-3.939\end{array}\right)\left(\begin{array}{c}1.018 x_{0}+0.449 l_{0}+1.743 \\
-0.060 x_{0}+0.150 l_{0}+0.095 \\
0.042 x_{0}-0.599 l_{0}-1.838\end{array}\right)$ \\
$1\left(\begin{array}{c}1.018 x_{1}-0.372 l_{1}+1.743 \\
-0.060 x_{1}+0.198 l_{1}+0.095 \\
0.042 x_{1}-0.633 l_{1}-1.838\end{array}\right)\left(\begin{array}{c}1.018 x_{1}+0.449 l_{1}+1.743 \\
-0.060 x_{1}+0.150 l_{1}+0.095 \\
0.042 x_{1}-0.599 l_{1}-1.838\end{array}\right)$ \\
\hline
\end{tabular}

\section{Numerical Illustration}

In this section, we present numerical examples to gain insights regarding the impact of time diversification and of liability on the optimal time-consistent strategies. To make it easy to analysis, we assume $T=2$ and all parameters at different periods are the same. Considering a market with three risky assets whose corresponding expected return vector and the variance-covariance matrices are given as $\mu_{t}=$ $(1.162,1.246,1.228)$ and

$$
\Sigma_{t}=\left(\begin{array}{lll}
0.0146 & 0.0187 & 0.0145 \\
0.0187 & 0.0854 & 0.0104 \\
0.0145 & 0.0104 & 0.0289
\end{array}\right),
$$

respectively. The expected return of the liability $E\left(Q_{t}\right)$ is 1.136, the corresponding variance $\operatorname{Var}\left(Q_{t}\right)$ is 0.01 , and the covariance vector $\Sigma_{t}^{0}$ is $(0.0006,0.0149,0.0050)^{\prime}$.

Table 3 illustrates how the time-consistent strategy depends on the liability. From Table 3, if an investor has a liability, then he/she could adjust their investment strategy which results in a parallel shift of the optimal time-consistent strategy. Thus, the investor should take account for the impact of liability.

Tables 4 and 5 show the time-consistent strategy and the myopic strategy in a market without riskless asset for $\lambda_{t}=$ 0.5 and $\lambda_{t}=0$, respectively. In Table 4 , we find that the two strategies are different and the difference between them enters into all of the three parts. Table 5 figures out that the two strategies are still very different even if the investor is arbitrarily risk averse. Further, Tables 4 and 5 imply that the investor can not ignore the time diversification effects in a market without riskless asset.

Next, we consider a market consisting of both risky assets and a riskless asset. Suppose that the return of the 
TABLE 5: Investment strategies in a market without riskless asset for $\lambda_{0}=\lambda_{1}=0$.

\begin{tabular}{lcc}
\hline$t$ & Time-consistent strategy $u_{t}^{*}$ & Myopic strategy $u_{t}^{m y}$ \\
\hline 0 & $\left(\begin{array}{c}0.903 x_{0}+1.194 l_{0} \\
-0.066 x_{0}+0.107 l_{0} \\
0.163 x_{0}-0.577 l_{0}\end{array}\right)$ & $\left(\begin{array}{c}1.018 x_{0}+0.449 l_{0} \\
-0.060 x_{0}+0.150 l_{0} \\
0.042 x_{0}-0.599 l_{0}\end{array}\right)$ \\
\hline 1 & $\left(\begin{array}{c}1.018 x_{1}-0.372 l_{1} \\
-0.060 x_{1}+0.198 l_{1} \\
0.042 x_{1}-0.633 l_{1}\end{array}\right)$ & $\left(\begin{array}{c}1.018 x_{1}+0.449 l_{1} \\
-0.060 x_{1}+0.150 l_{1} \\
0.042 x_{1}-0.599 l_{1}\end{array}\right)$ \\
\hline
\end{tabular}

TABLE 6: Investment strategies in a market with a riskless asset for $\lambda_{0}=\lambda_{1}=0.5$.

\begin{tabular}{lcc}
\hline$t$ & Time-consistent strategy $u_{t}^{*}$ & Myopic strategy $u_{t}^{m y}$ \\
\hline & $\left(\begin{array}{c}0.444 l_{0}+7.889 \\
0.150 l_{0}-0.062 \\
-0.599 l_{0}-3.572\end{array}\right)$ & $\left(\begin{array}{c}0.444 l_{0}+3.867 \\
0.150 l_{0}-0.031 \\
-0.599 l_{0}-1.751\end{array}\right)$ \\
1 & $\left(\begin{array}{c}0.444 l_{1}+3.867 \\
0.150 l_{1}-0.031 \\
-0.599 l_{1}-1.751\end{array}\right)$ & $\left(\begin{array}{c}0.444 l_{1}+3.867 \\
0.150 l_{1}-0.031 \\
-0.599 l_{1}-1.751\end{array}\right)$ \\
\hline
\end{tabular}

TABLE 7: Investment strategies in a market with a riskless asset for $\lambda_{0}=\lambda_{1}=0$.

\begin{tabular}{ccc}
\hline$t$ & Time-consistent strategy $u_{t}^{*}$ & Myopic strategy $u_{t}^{m y}$ \\
0 & $\left(\begin{array}{c}0.444 l_{0} \\
0.150 l_{0} \\
-0.599 l_{0}\end{array}\right)$ & $\left(\begin{array}{c}0.444 l_{0} \\
0.150 l_{0} \\
-0.599 l_{0}\end{array}\right)$ \\
1 & $\left(\begin{array}{c}0.444 l_{1} \\
0.150 l_{1} \\
-0.599 l_{1}\end{array}\right)$ & $\left(\begin{array}{c}0.444 l_{1} \\
0.150 l_{1} \\
-0.599 l_{1}\end{array}\right)$ \\
\hline
\end{tabular}

riskless asset $s_{t}$ is 1.04 . Then we have $\epsilon_{t}=\mu_{t}-s_{t} I=$ $(0.122,0.206,0.188)^{\prime}$ and $\varphi_{t}=\Sigma_{t}^{0}=(0.0006,0.0149,0.0050)^{\prime}$.

Tables 6 and 7 show the time-consistent strategy and the myopic strategy in a market with a riskless asset for $\lambda_{t}=0.5$ and $\lambda_{t}=0$, respectively. From Table 6 , it is clear that the two strategies are different which is consistent with the results in a market without riskless asset. However, they may be exactly the same if the investor is arbitrarily risk averse. This implies that the investor who is arbitrarily risk averse can ignore the time diversification effects.

\section{Conclusion}

In this paper, we consider the time-consistency of the optimal asset-liability management policies in a market with and without a riskless asset, respectively. By employing the dynamic programming technique, we give the optimal timeconsistent investment policies. After comparing the optimal time-consistent policies with myopic strategies, we show that, a risk averse investor should concern the time diversification effects. Further, an arbitrarily risk averse investor could ignore these effects in a market with a riskless asset.

\section{Appendices}

\section{A. Proof of $(20)$}

Firstly, substituting $u_{T-1}^{*}$ into ${u_{T-1}^{*}}^{\prime} \Sigma_{T-1}^{1} u_{T-1}^{*}$ gives

$$
\begin{aligned}
u_{T-1}^{*}{ }^{\prime} \Sigma_{T-1}^{1} u_{T-1}^{*}= & \left\{\frac{I}{a_{T-1}} x_{T-1}+\left[\Sigma_{T-1}^{0}-\gamma_{T-1} I\right] l_{T-1}\right. \\
& \left.+\frac{\lambda_{T-1}}{2}\left[\mu_{T-1}-q_{T-1} I\right]\right\}^{\prime}\left(\Sigma_{T-1}^{1}\right)^{-1} \\
& \times\left\{\frac{I}{a_{T-1}} x_{T-1}+\left[\Sigma_{T-1}^{0}-\gamma_{T-1} I\right]\right. \\
= & \frac{1}{a_{T-1}} x_{T-1}^{2}+\left[l_{T-1}^{0}+\frac{\lambda_{T-1}}{2}\left[\mu_{T-1}-q_{T-1} I\right]^{\prime}\right] \\
& \times\left(\Sigma_{T-1}^{1}\right)^{-1}\left[\Sigma_{T-1}^{0}-\gamma_{T-1} I\right] l_{T-1}^{2} \\
& +\lambda_{T-1}\left[\Sigma_{T-1}^{0}-\gamma_{T-1} I\right]^{\prime} \\
& \times\left(\Sigma_{T-1}^{1}\right)^{-1}\left[\mu_{T-1}-q_{T-1} I\right] l_{T-1} \\
& +\frac{\left(\lambda_{T-1}\right)^{2}}{4}\left[\mu_{T-1}-q_{T-1} I\right]^{\prime} \\
& \times\left(\Sigma_{T-1}^{1}\right)^{-1}\left[\mu_{T-1}-q_{T-1} I\right] \\
= & \frac{1}{a_{T-1}} x_{T-1}^{2}+\left[\left(\Sigma_{T-1}^{0}\right)^{\prime}\left(\Sigma_{T-1}^{1}\right)^{-1} \Sigma_{T-1}^{0}\left(\Sigma_{T-1}^{1}\right)^{-1} \mu_{T-1}-b_{T-1} \gamma_{T-1}\right] \\
& \frac{\left(\lambda_{T-1}\right)^{2}}{4} \alpha_{T-1} . \\
& \left.+a_{T-1} \gamma_{T-1}^{2}\right] l_{T-1}^{2} \\
& +A
\end{aligned}
$$

Secondly, by substituting $u_{T-1}^{*}$ into $2\left[\left(\Sigma_{T-1}^{0}\right)^{\prime} l_{T-1}+\right.$ $\left.\left(\lambda_{T-1} / 2\right) \mu_{T-1}^{\prime}\right] u_{T-1}^{*}$, we have

$$
\begin{aligned}
& 2\left[\left(\Sigma_{T-1}^{0}\right)^{\prime} l_{T-1}+\frac{\lambda_{T-1}}{2} \mu_{T-1}^{\prime}\right] u_{T-1}^{*} \\
& =\frac{2}{a_{T-1}}\left[\left(\Sigma_{T-1}^{0}\right)^{\prime} l_{T-1}+\frac{\lambda_{T-1}}{2} \mu_{T-1}^{\prime}\right]\left(\Sigma_{T-1}^{1}\right)^{-1} I x_{T-1} \\
& \quad+2\left[\left(\Sigma_{T-1}^{0}\right)^{\prime} l_{T-1}+\frac{\lambda_{T-1}}{2} \mu_{T-1}^{\prime}\right]\left(\Sigma_{T-1}^{1}\right)^{-1}
\end{aligned}
$$




$$
\begin{aligned}
& \times\left[\Sigma_{T-1}^{0}-\gamma_{T-1} I\right] l_{T-1} \\
& +\lambda_{T-1}\left[\left(\Sigma_{T-1}^{0}\right)^{\prime} l_{T-1}+\frac{\lambda_{T-1}}{2} \mu_{T-1}^{\prime}\right]\left(\Sigma_{T-1}^{1}\right)^{-1} \\
& \times\left[\mu_{T-1}-q_{T-1} I\right] \\
= & 2 \gamma_{T-1} l_{T-1} x_{T-1}+q_{T-1} \lambda_{T-1} x_{T-1} \\
& +2\left[\left(\Sigma_{T-1}^{0}\right)^{\prime}\left(\Sigma_{T-1}^{1}\right)^{-1} \Sigma_{T-1}^{0}-\gamma_{T-1} d_{T-1}\right] l_{T-1}^{2} \\
& +2 \lambda_{T-1}\left[\left(\Sigma_{T-1}^{0}\right)^{\prime}\left(\Sigma_{T-1}^{1}\right)^{-1} \mu_{T-1}-b_{T-1} \gamma_{T-1}\right] l_{T-1} \\
& +\frac{\left(\lambda_{T-1}\right)^{2}}{2} \alpha_{T-1} .
\end{aligned}
$$

Thus,

$$
\begin{aligned}
& V_{T-1}^{*}\left(x_{T-1}, l_{T-1}\right) \\
& =\frac{1}{a_{T-1}} x_{T-1}^{2}-\lambda_{T-1} q_{T-1} x_{T-1}-2 \gamma_{T-1} x_{T-1} l_{T-1} \\
& +\lambda_{T-1}\left[E\left(Q_{T-1}\right)-\left(\Sigma_{T-1}^{0}\right)^{\prime}\left(\Sigma_{T-1}^{1}\right)^{-1} \mu_{T-1}\right. \\
& \left.\quad+b_{T-1} \gamma_{T-1}\right] l_{T-1} \\
& +\quad\left[\operatorname{Var}\left(Q_{T-1}\right)-\left(\Sigma_{T-1}^{0}\right)^{\prime}\left(\Sigma_{T-1}^{1}\right)^{-1} \Sigma_{T-1}^{0}\right. \\
& \left.\quad+\gamma_{T-1} d_{T-1}\right] l_{T-1}^{2}-\frac{\left(\lambda_{T-1}\right)^{2}}{4} \alpha_{T-1} \\
& =\frac{1}{a_{T-1}} x_{T-1}^{2}-\lambda_{T-1} q_{T-1} x_{T-1}-2 \gamma_{T-1} x_{T-1} l_{T-1} \\
& \quad+\beta_{1, T-1} l_{T-1}+\beta_{2, T-1} l_{T-1}^{2}-\frac{\left(\lambda_{T-1}\right)^{2}}{4} \alpha_{T-1} .
\end{aligned}
$$

\section{B. Proof of $(34)$}

It is easy to verify that

$$
\begin{aligned}
& \frac{I^{\prime}}{a_{T-2}}\left(\Sigma_{T-2}^{1}\right)^{-1}\left[\Sigma_{T-2}^{0}+\gamma_{T-1} E\left(Q_{T-2} R_{T-2}\right)-\widetilde{\gamma}_{T-2} I\right] \\
& \quad=\frac{d_{T-2}}{a_{T-2}}+\gamma_{T-1} \frac{I^{\prime}\left(\Sigma_{T-2}^{1}\right)^{-1} E\left(Q_{T-2} R_{T-2}\right)}{a_{T-2}}-\widetilde{\gamma}_{T-2} \\
& \quad=\gamma_{T-2}+\gamma_{T-1} \frac{I^{\prime}\left(\Sigma_{T-2}^{1}\right)^{-1} E\left(Q_{T-2} R_{T-2}\right)}{a_{T-2}}-\widetilde{\gamma}_{T-2} \\
& =\widetilde{\gamma}_{T-2}-\widetilde{\gamma}_{T-2} \\
& =0, \\
& \frac{I^{\prime}}{a_{T-2}}\left(\Sigma_{T-2}^{1}\right)^{-1}\left[\mu_{T-2}-q_{T-2} I\right]=\frac{b_{T-2}}{a_{T-2}}-q_{T-2}=0, \\
& \quad\left[\Sigma_{T-2}^{0}+\gamma_{T-1} E\left(Q_{T-2} R_{T-2}\right)\right]^{\prime}\left(\Sigma_{T-2}^{1}\right)^{-1} I
\end{aligned}
$$

$$
\begin{aligned}
& =a_{T-2}\left\{\gamma_{T-2}+\gamma_{T-1} \frac{E\left(Q_{T-2} R_{T-2}^{\prime}\right)\left(\Sigma_{T-2}^{1}\right)^{-1} I}{a_{T-2}}\right\} \\
& =a_{T-2} \widetilde{\gamma}_{T-2} .
\end{aligned}
$$

Firstly, we compute $u_{T-2}^{*}{ }^{\prime} \Sigma_{T-2}^{1} u_{T-2}^{*}$. It follows from the previous equalities that both coefficients of $x_{T-2}$ and $l_{T-2} x_{T-2}$ in $u_{T-2}^{*} \Sigma_{T-2}^{1} u_{T-2}^{*}$ are 0 ; the coefficient of $x_{T-2}^{2}$ is

$$
\frac{I^{\prime}}{a_{T-2}}\left(\Sigma_{T-2}^{1}\right)^{-1} \frac{I}{a_{T-2}}=\frac{1}{a_{T-2}}
$$

the coefficient of $l_{T-2}$ is

$$
\lambda_{T-2}^{1}\left[\Sigma_{T-2}^{0}+\gamma_{T-1} E\left(Q_{T-2} R_{T-2}\right)-\widetilde{\gamma}_{T-2} I\right]^{\prime}
$$

$$
\begin{aligned}
& \times\left(\Sigma_{T-2}^{1}\right)^{-1}\left[\mu_{T-2}-q_{T-2} I\right] \\
= & \lambda_{T-2}^{1}\left[\Sigma_{T-2}^{0}+\gamma_{T-1} E\left(Q_{T-2} R_{T-2}\right)\right]^{\prime} \\
& \times\left(\Sigma_{T-2}^{1}\right)^{-1}\left[\mu_{T-2}-q_{T-2} I\right] \\
& -\lambda_{T-2}^{1} \widetilde{\gamma}_{T-2} I^{\prime}\left(\Sigma_{T-2}^{1}\right)^{-1}\left[\mu_{T-2}-q_{T-2} I\right] \\
= & \lambda_{T-2}^{1}\left[\Sigma_{T-2}^{0}+\gamma_{T-1} E\left(Q_{T-2} R_{T-2}\right)\right]^{\prime} \\
& \times\left(\Sigma_{T-2}^{1}\right)^{-1}\left[\mu_{T-2}-q_{T-2} I\right],
\end{aligned}
$$

the coefficient of $l_{T-2}^{2}$ is

$$
\begin{aligned}
{\left[\Sigma_{T-2}^{0}+\right.} & \left.\gamma_{T-1} E\left(Q_{T-2} R_{T-2}\right)-\tilde{\gamma}_{T-2} I\right]^{\prime} \\
& \cdot\left(\Sigma_{T-2}^{1}\right)^{-1} \\
& \cdot\left[\Sigma_{T-2}^{0}+\gamma_{T-1} E\left(Q_{T-2} R_{T-2}\right)-\widetilde{\gamma}_{T-2} I\right] \\
= & {\left[\Sigma_{T-2}^{0}+\gamma_{T-1} E\left(Q_{T-2} R_{T-2}\right)\right]^{\prime} } \\
& \times\left(\Sigma_{T-2}^{1}\right)^{-1}\left[\Sigma_{T-2}^{0}+\gamma_{T-1} E\left(Q_{T-2} R_{T-2}\right)\right] \\
& -a_{T-2} \tilde{\gamma}_{T-2}^{2},
\end{aligned}
$$

and the constant term is

$$
\begin{aligned}
& \frac{\left(\lambda_{T-2}^{1}\right)^{2}}{4}\left[\mu_{T-2}-q_{T-2} I\right]^{\prime}\left(\Sigma_{T-2}^{1}\right)^{-1}\left[\mu_{T-2}-q_{T-2} I\right] \\
& \quad=\frac{\left(\lambda_{T-2}^{1}\right)^{2}}{4} \alpha_{T-2} .
\end{aligned}
$$

Secondly, we compute $\Phi_{T-2}^{\prime} u_{T-2}^{*}$. The coefficient of $x_{T-2}^{2}$ is 0 , the coefficient of term $x_{T-2}$ is

$$
\frac{\lambda_{T-2}^{1}}{2} \frac{\mu_{T-2}^{\prime}\left(\Sigma_{T-2}^{1}\right)^{-1} I}{a_{T-2}}=\frac{\lambda_{T-2}^{1} q_{T-2}}{2},
$$


the coefficient of $l_{T-2} x_{T-2}$ is $\tilde{\gamma}_{T-2}$, the coefficient of $l_{T-2}$ is

$$
\begin{aligned}
\frac{\lambda_{T-2}^{1}}{2}\left[\Sigma_{T-2}^{0}+\gamma_{T-1} E\left(Q_{T-2} R_{T-2}\right)\right]^{\prime} \\
\quad \times\left(\Sigma_{T-2}^{1}\right)^{-1}\left(\mu_{T-2}-q_{T-2} I\right) \\
\quad+\frac{\lambda_{T-2}^{1}}{2} \mu_{T-2}^{\prime}\left(\Sigma_{T-2}^{1}\right)^{-1} \\
\quad \times\left[\Sigma_{T-2}^{0}+\gamma_{T-1} E\left(Q_{T-2} R_{T-2}\right)-\widetilde{\gamma}_{T-2} I\right],
\end{aligned}
$$

the coefficient of $l_{T-2}^{2}$ is

$$
\begin{aligned}
& {\left[\Sigma_{T-2}^{0}+\gamma_{T-1} E\left(Q_{T-2} R_{T-2}\right)\right]^{\prime}\left(\Sigma_{T-2}^{1}\right)^{-1}} \\
& \quad \times\left[\Sigma_{T-2}^{0}+\gamma_{T-1} E\left(Q_{T-2} R_{T-2}\right)-\widetilde{\gamma}_{T-2} I\right] \\
& =\left[\Sigma_{T-2}^{0}+\gamma_{T-1} E\left(Q_{T-2} R_{T-2}\right)\right]^{\prime}\left(\Sigma_{T-2}^{1}\right)^{-1} \\
& \quad \times\left[\Sigma_{T-2}^{0}+\gamma_{T-1} E\left(Q_{T-2} R_{T-2}\right)\right]-a_{T-2} \widetilde{\gamma}_{T-2}^{2},
\end{aligned}
$$

and the constant term is

$$
\left(\frac{\lambda_{T-2}^{1}}{2}\right)^{2} \mu_{T-2}^{\prime}\left(\Sigma_{T-2}^{1}\right)^{-1}\left(\mu_{T-2}-q_{T-2} I\right)=\left(\frac{\lambda_{T-2}^{1}}{2}\right)^{2} \alpha_{T-2} .
$$

Then we can compute $V_{T-2}^{*}\left(x_{T-2}, l_{T-2}\right)$ easily. The coefficient of $x_{T-2}^{2}$ is

$$
\frac{I^{\prime}}{a_{T-2}}\left(\Sigma_{T-2}^{1}\right)^{-1} \frac{I}{a_{T-2}}=\frac{1}{a_{T-2}},
$$

the coefficient of $x_{T-2}$ is $-\lambda_{T-2}^{1} q_{T-2}$, the coefficient of $l_{T-2} x_{T-2}$ is $-2 \widetilde{\gamma}_{T-2}$, the coefficient of $l_{T-2}^{2}$ is

$$
\begin{aligned}
& {\left[\operatorname{Var}\left(Q_{T-2}\right)+\beta_{2, T-1} E\left(Q_{T-2}^{2}\right)\right]+a_{T-2} \widetilde{\gamma}_{T-2}^{2}} \\
& \quad-\left[\Sigma_{T-2}^{0}+\gamma_{T-1} E\left(Q_{T-2} R_{T-2}\right)\right]^{\prime}\left(\Sigma_{T-2}^{1}\right)^{-1} \\
& \quad \times\left[\Sigma_{T-2}^{0}+\gamma_{T-1} E\left(Q_{T-2} R_{T-2}\right)\right] \\
& =\beta_{2, T-2},
\end{aligned}
$$

the coefficient of $l_{T-2}$ is

$$
\begin{aligned}
- & \lambda_{T-2}^{1} \mu_{T-2}^{\prime}\left(\Sigma_{T-2}^{1}\right)^{-1} \\
& \times\left[\Sigma_{T-2}^{0}+\gamma_{T-1} E\left(Q_{T-2} R_{T-2}\right)-\widetilde{\gamma}_{T-2} I\right] \\
& +\left[\lambda_{T-2} E\left(Q_{T-2}\right)+\beta_{1, T-1} E\left(Q_{T-2}\right)\right] \\
= & \beta_{1, T-2},
\end{aligned}
$$

and the constant term is

$$
-\frac{\lambda_{T-1}^{2}}{4} \alpha_{T-1}-\frac{\left(\lambda_{T-2}^{1}\right)^{2}}{4} \alpha_{T-2} .
$$

Thus,

$$
\begin{aligned}
V_{T-2}^{*}\left(x_{T-2}, l_{T-2}\right)= & \frac{1}{a_{T-2}} x_{T-2}^{2}-\lambda_{T-2}^{1} q_{T-2} x_{T-2} \\
& -2 \widetilde{\gamma}_{T-2} x_{T-2} l_{T-2}+\beta_{1, T-2} l_{T-2} \\
& +\beta_{2, T-2} l_{T-2}^{2}-\frac{\lambda_{T-1}^{2}}{4} \alpha_{T-1}-\frac{\left(\lambda_{T-2}^{1}\right)^{2}}{4} \alpha_{T-2} .
\end{aligned}
$$

\section{Proof of $(46)$}

It is easy to verify that

$$
\begin{aligned}
\frac{I^{\prime}}{a_{t-1}}\left(\Sigma_{t-1}^{1}\right)^{-1}\left[\Sigma_{t-1}^{0}+\widetilde{\gamma}_{t} E\left(Q_{t-1} R_{t-1}\right)-\widetilde{\gamma}_{t-1} I\right] \\
=\frac{d_{t-1}}{a_{t-1}}+\widetilde{\gamma}_{t} \frac{I^{\prime}\left(\Sigma_{t-1}^{1}\right)^{-1} E\left(Q_{t-1} R_{t-1}\right)}{a_{t-1}}-\widetilde{\gamma}_{t-1} \\
=\gamma_{t-1}+\widetilde{\gamma}_{t} \frac{I^{\prime}\left(\Sigma_{t-1}^{1}\right)^{-1} E\left(Q_{t-1} R_{t-1}\right)}{a_{t-1}}-\widetilde{\gamma}_{t-1} \\
=\widetilde{\gamma}_{t-1}-\widetilde{\gamma}_{t-1} \\
=0,
\end{aligned}
$$

$$
\begin{aligned}
& \frac{I^{\prime}}{a_{t-1}}\left(\Sigma_{t-1}^{1}\right)^{-1}\left[\mu_{t-1}-q_{t-1} I\right]=\frac{b_{t-1}}{a_{t-1}}-q_{t-1}=0, \\
& {\left[\Sigma_{t-1}^{0}+\widetilde{\gamma}_{t} E\left(Q_{t-1} R_{t-1}\right)\right]^{\prime}\left(\Sigma_{t-1}^{1}\right)^{-1} I} \\
& =a_{t-1}\left\{\gamma_{t-1}+\widetilde{\gamma}_{t} \frac{E\left(Q_{t-1} R_{t-1}^{\prime}\right)\left(\Sigma_{t-1}^{1}\right)^{-1} I}{a_{t-1}}\right\} \\
& =a_{t-1} \widetilde{\gamma}_{t-1} .
\end{aligned}
$$

Firstly, we compute $u_{t-1}^{*}{ }^{\prime} \Sigma_{t-1}^{1} u_{t-1}^{*}$. It follows from the previous equalities that both coefficients of $x_{t-1}$ and $l_{t-1} x_{t-1}$ are 0 ; the coefficient of $x_{t-1}^{2}$ is

$$
\frac{I^{\prime}}{a_{t-1}}\left(\Sigma_{t-1}^{1}\right)^{-1} \frac{I}{a_{t-1}}=\frac{1}{a_{t-1}},
$$

the coefficient of $l_{t-1}$ is

$$
\begin{aligned}
\lambda_{t-1}^{1}\left[\Sigma_{t-1}^{0}\right. & \left.+\widetilde{\gamma}_{t} E\left(Q_{t-1} R_{t-1}\right)-\widetilde{\gamma}_{t-1} I\right]^{\prime} \\
& \times\left(\Sigma_{t-1}^{1}\right)^{-1}\left[\mu_{t-1}-q_{t-1} I\right] \\
= & \lambda_{t-1}^{1}\left[\Sigma_{t-1}^{0}+\widetilde{\gamma}_{t} E\left(Q_{t-1} R_{t-1}\right)\right]^{\prime} \\
& \times\left(\Sigma_{t-1}^{1}\right)^{-1}\left[\mu_{t-1}-q_{t-1} I\right] \\
& -\lambda_{t-1}^{1} \widetilde{\gamma}_{t-1} I^{\prime}\left(\Sigma_{t-1}^{1}\right)^{-1}\left[\mu_{t-1}-q_{t-1} I\right]
\end{aligned}
$$




$$
\begin{aligned}
= & \lambda_{t-1}^{1}\left[\Sigma_{t-1}^{0}+\widetilde{\gamma}_{t} E\left(Q_{t-1} R_{t-1}\right)\right]^{\prime} \\
& \times\left(\Sigma_{t-1}^{1}\right)^{-1}\left[\mu_{t-1}-q_{t-1} I\right],
\end{aligned}
$$

the coefficient of $l_{T-2}^{2}$ is

$$
\begin{aligned}
& {\left[\Sigma_{t-1}^{0}+\widetilde{\gamma}_{t} E\left(Q_{t-1} R_{t-1}\right)-\widetilde{\gamma}_{t-1} I\right]^{\prime} \cdot\left(\Sigma_{t-1}^{1}\right)^{-1}} \\
& \cdot\left[\Sigma_{t-1}^{0}+\widetilde{\gamma}_{t} E\left(Q_{t-1} R_{t-1}\right)-\widetilde{\gamma}_{t-1} I\right] \\
& =\left[\Sigma_{t-1}^{0}+\widetilde{\gamma}_{t} E\left(Q_{t-1} R_{t-1}\right)\right]^{\prime}\left(\Sigma_{t-1}^{1}\right)^{-1} \\
& \quad \times\left[\Sigma_{t-1}^{0}+\widetilde{\gamma}_{t} E\left(Q_{t-1} R_{t-1}\right)\right]-a_{t-1} \widetilde{\gamma}_{t-1}^{2},
\end{aligned}
$$

and the constant term is

$$
\frac{\left(\lambda_{t-1}^{1}\right)^{2}}{4}\left[\mu_{t-1}-q_{t-1} I\right]^{\prime}\left(\Sigma_{t-1}^{1}\right)^{-1}\left[\mu_{t-1}-q_{t-1} I\right]=\frac{\left(\lambda_{t-1}^{1}\right)^{2}}{4} \alpha_{t-1} \text {. }
$$

Secondly, we compute $\Phi_{t-1}^{\prime} u_{t-1}^{*}$. The coefficient of $x_{t-1}^{2}$ is 0 , the coefficient of $x_{t-1}$ is

$$
\frac{\lambda_{t-1}^{1}}{2} \frac{\mu_{t-1}^{\prime}\left(\Sigma_{t-1}^{1}\right)^{-1} I}{a_{t-1}}=\frac{\lambda_{t-1}^{1} q_{t-1}}{2},
$$

the coefficient of $l_{t-1} x_{t-1}$ is $\widetilde{\gamma}_{t-1}$, the coefficient of $l_{t-1}$ is

$$
\begin{aligned}
\frac{\lambda_{t-1}^{1}}{2}\left[\Sigma_{t-1}^{0}\right. & \left.+\widetilde{\gamma}_{t} E\left(Q_{t-1} R_{t-1}\right)\right]^{\prime}\left(\Sigma_{t-1}^{1}\right)^{-1} \\
& \times\left(\mu_{t-1}-q_{t-1} I\right)+\frac{\lambda_{t-1}^{1}}{2} \mu_{t-1}^{\prime}\left(\Sigma_{t-1}^{1}\right)^{-1} \\
& \times\left[\Sigma_{t-1}^{0}+\widetilde{\gamma}_{t} E\left(Q_{t-1} R_{t-1}\right)-\widetilde{\gamma}_{t-1} I\right]
\end{aligned}
$$

the coefficient of $l_{t-1}^{2}$ is

$$
\begin{aligned}
& {\left[\Sigma_{t-1}^{0}+\widetilde{\gamma}_{t} E\left(Q_{t-1} R_{t-1}\right)\right]^{\prime}\left(\Sigma_{t-1}^{1}\right)^{-1}} \\
& \quad \times\left[\Sigma_{t-1}^{0}+\widetilde{\gamma}_{t} E\left(Q_{t-1} R_{t-1}\right)-\widetilde{\gamma}_{t-1} I\right] \\
& =\left[\Sigma_{t-1}^{0}+\widetilde{\gamma}_{t} E\left(Q_{t-1} R_{t-1}\right)\right]^{\prime}\left(\Sigma_{t-1}^{1}\right)^{-1} \\
& \quad \times\left[\Sigma_{t-1}^{0}+\widetilde{\gamma}_{t} E\left(Q_{t-1} R_{t-1}\right)\right]-a_{t-1} \widetilde{\gamma}_{t-1}^{2},
\end{aligned}
$$

and the constant term is

$$
\left(\frac{\lambda_{t-1}^{1}}{2}\right)^{2} \mu_{t-1}\left(\Sigma_{t-1}^{1}\right)^{-1}\left(\mu_{t-1}-q_{t-1} I\right)=\left(\frac{\lambda_{t-1}^{1}}{2}\right)^{2} \alpha_{t-1} .
$$

Then it is easy to compute $V_{t-1}^{*}\left(x_{t-1}, l_{t-1}\right)$. The coefficient of $x_{t-1}^{2}$ is $1 / a_{t-1}$, the coefficient of $x_{t-1}$ is $-\lambda_{t-1}^{1} q_{t-1}$, the coefficient of $l_{t-1} x_{t-1}$ is $-2 \widetilde{\gamma}_{t-1}$, the coefficient of $l_{t-1}^{2}$ is

$$
\begin{aligned}
& {\left[\operatorname{Var}\left(Q_{t-1}\right)+\beta_{2, t} E\left(Q_{t-1}^{2}\right)\right]+a_{t-1} \widetilde{\gamma}_{t-1}^{2}} \\
& \quad-\left[\Sigma_{t-1}^{0}+\widetilde{\gamma}_{t} E\left(Q_{t-1} R_{t-1}\right)\right]^{\prime}\left(\Sigma_{t-1}^{1}\right)^{-1} \\
& \quad \times\left[\Sigma_{t-1}^{0}+\widetilde{\gamma}_{t} E\left(Q_{t-1} R_{t-1}\right)\right] \\
& =\beta_{2, t-1},
\end{aligned}
$$

the coefficient of $l_{t-1}$ is

$$
\begin{aligned}
& -\lambda_{t-1}^{1} \mu_{t-1}^{\prime}\left(\Sigma_{t-1}^{1}\right)^{-1}\left[\Sigma_{t-1}^{0}+\widetilde{\gamma}_{t} E\left(Q_{t-1} R_{t-1}\right)-\widetilde{\gamma}_{t-1} I\right] \\
& +\left(\lambda_{t-1}+\beta_{1, t}\right) E\left(Q_{t-1}\right)=\beta_{1, t-1},
\end{aligned}
$$

and the constant term is $-(1 / 4) \sum_{i=t-1}^{T-1}\left(\lambda_{i}^{1}\right)^{2} \alpha_{i}$.

Thus,

$$
\begin{aligned}
V_{t-1}^{*}\left(x_{t-1}, l_{t-1}\right)= & \frac{1}{a_{t-1}} x_{t-1}^{2}-\lambda_{t-1}^{1} q_{t-1} x_{t-1} \\
& -2 \widetilde{\gamma}_{t-1} x_{t-1} l_{t-1}+\beta_{1, t-1} l_{t-1} \\
& +\beta_{2, t-1} l_{t-1}^{2}-\frac{1}{4} \sum_{i=t-1}^{T-1}\left(\lambda_{i}^{1}\right)^{2} \alpha_{i} .
\end{aligned}
$$

\section{Proof of (62)}

Substituting $u_{T-1}^{*}$ into $u_{T-1}^{*}{ }^{\prime} \Sigma_{T-1} u_{T-1}^{*}$, we have

$$
\begin{aligned}
u_{T-1}^{*} \Sigma_{T-1} u_{T-1}^{*}= & \left(\varphi_{T-1}^{\prime} l_{T-1}+\frac{\lambda_{T-1}}{2} \epsilon_{T-1}^{\prime}\right) \\
& \times\left(\Sigma_{T-1}\right)^{-1}\left(\varphi_{T-1} l_{T-1}+\frac{\lambda_{T-1}}{2} \epsilon_{T-1}\right) \\
= & \varphi_{T-1}^{\prime}\left(\Sigma_{T-1}\right)^{-1} \varphi_{T-1} l_{T-1}^{2}+\lambda_{T-1} \epsilon_{T-1}^{\prime} \\
& \times\left(\Sigma_{T-1}\right)^{-1} \varphi_{T-1} l_{T-1}+\frac{\left(\lambda_{T-1}\right)^{2}}{4} M_{T-1},
\end{aligned}
$$

$$
2\left(\varphi_{T-1}^{\prime} l_{T-1}+\frac{\lambda_{T-1}}{2} \epsilon_{T-1}^{\prime}\right) u_{T-1}^{*}=2 u_{T-1}^{*}{ }^{\prime} \Sigma_{T-1} u_{T-1}^{*} .
$$

Thus,

$$
\begin{aligned}
V_{T-1}^{*}\left(x_{T-1}, l_{T-1}\right)= & -u_{T-1}^{*}{ }^{\prime} \Sigma_{T-1} u_{T-1}^{*}+\operatorname{Var}\left(Q_{T-1}\right) l_{T-1}^{2} \\
& -\lambda_{T-1} s_{T-1} x_{T-1}+\lambda_{T-1} E\left(Q_{T-1}\right) l_{T-1} \\
= & {\left[\operatorname{Var}\left(Q_{T-1}\right)-\varphi_{T-1}^{\prime}\left(\Sigma_{T-1}\right)^{-1} \varphi_{T-1}\right] l_{T-1}^{2} } \\
& +\lambda_{T-1}\left[E\left(Q_{T-1}\right)-\epsilon_{T-1}^{\prime}\left(\Sigma_{T-1}\right)^{-1} \varphi_{T-1}\right] \\
& \times l_{T-1}-\lambda_{T-1} s_{T-1} x_{T-1}-\frac{\left(\lambda_{T-1}\right)^{2}}{4} M_{T-1} \\
= & \bar{\beta}_{2, T-1} l_{T-1}^{2}+\bar{\beta}_{1, T-1} l_{T-1} \\
& -\lambda_{T-1} s_{T-1} x_{T-1}-\frac{\left(\lambda_{T-1}\right)^{2}}{4} M_{T-1} .
\end{aligned}
$$




\section{E. Proof of (69)}

Taking $u_{T-2}^{*}$ into account, we have

$$
\begin{aligned}
u_{T-2}^{*}{ }^{\prime} \Sigma_{T-2} u_{T-2}^{*}= & \left(\varphi_{T-2}^{\prime} l_{T-2}+\frac{\lambda_{T-2}^{1}}{2} \epsilon_{T-2}^{\prime}\right) \\
& \times\left(\Sigma_{T-2}\right)^{-1}\left(\varphi_{T-2} l_{T-2}+\frac{\lambda_{T-2}^{1}}{2} \epsilon_{T-2}\right) \\
= & \varphi_{T-2}^{\prime}\left(\Sigma_{T-2}\right)^{-1} \varphi_{T-2} l_{T-2}^{2}+\lambda_{T-2}^{1} \epsilon_{T-2}^{\prime} \\
& \times\left(\Sigma_{T-2}\right)^{-1} \varphi_{T-2} l_{T-2}+\frac{\left(\lambda_{T-2}^{1}\right)^{2}}{4} M_{T-2}, \\
2\left\{\varphi_{T-2}^{\prime} l_{T-2}+\right. & \left.\frac{\lambda_{T-2}^{1}}{2} \epsilon_{T-2}^{\prime}\right\} u_{T-2}^{*}=2 u_{T-2}^{*} \Sigma_{T-2} u_{T-2}^{*} .
\end{aligned}
$$

Thus,

$$
\begin{aligned}
V_{T-2}^{*}\left(x_{T-2}, l_{T-2}\right)= & -u_{T-2}^{*}{ }^{\prime} \Sigma_{T-2} u_{T-2}^{*} \\
& +\left[\operatorname{Var}\left(Q_{T-2}\right)+\bar{\beta}_{2, T-1} E\left(Q_{T-2}^{2}\right)\right] l_{T-2}^{2} \\
& +\left[\lambda_{T-2} E\left(Q_{T-2}\right)+\bar{\beta}_{1, T-1} E\left(Q_{T-2}\right)\right] l_{T-1} \\
& -\lambda_{T-2}^{1} s_{T-2} x_{T-2}-\frac{\left(\lambda_{T-1}\right)^{2}}{4} M_{T-1} \\
= & \bar{\beta}_{2, T-2} l_{T-2}^{2}+\bar{\beta}_{1, T-2} l_{T-2}-\lambda_{T-2}^{1} s_{T-2} x_{T-2} \\
& -\frac{\left(\lambda_{T-1}\right)^{2}}{4} M_{T-1}-\frac{\left(\lambda_{T-2}^{1}\right)^{2}}{4} M_{T-2},
\end{aligned}
$$

where

$$
\begin{aligned}
& \bar{\beta}_{1, T-2}=\left(\lambda_{T-2}+\bar{\beta}_{1, T-1}\right) E\left(Q_{T-2}\right)-\lambda_{T-2}^{1} \epsilon_{T-2}^{\prime}\left(\Sigma_{T-2}\right)^{-1} \varphi_{T-2}, \\
& \beta_{2, T-2}=\operatorname{Var}\left(Q_{T-2}\right)+\bar{\beta}_{2, T-1} E\left(Q_{T-2}^{2}\right)-\varphi_{T-2}^{\prime}\left(\Sigma_{T-2}\right)^{-1} \varphi_{T-2} .
\end{aligned}
$$

\section{F. Proof of (77)}

It follows from $u_{t-1}^{*}$ that

$$
\begin{aligned}
u_{t-1}^{*}{ }^{\prime} \Sigma_{t-1} u_{t-1}^{*}= & \left(\varphi_{T-1}^{\prime} l_{t-1}+\frac{\lambda_{t-1}^{1}}{2} \epsilon_{T-1}^{\prime}\right) \\
& \times\left(\Sigma_{t-1}\right)^{-1}\left(\varphi_{t-1} l_{t-1}+\frac{\lambda_{t-1}^{1}}{2} \epsilon_{t-1}\right) \\
= & \varphi_{T-1}^{\prime}\left(\Sigma_{t-1}\right)^{-1} \varphi_{t-1} l_{t-1}^{2}+\lambda_{t-1}^{1} \epsilon_{T-1}^{\prime} \\
& \times\left(\Sigma_{t-1}\right)^{-1} \varphi_{t-1} l_{t-1}+\frac{\left(\lambda_{t-1}^{1}\right)^{2}}{4} M_{t-1}, \\
2\left(\varphi_{T-1}^{\prime} l_{t-1}+\right. & \left.\frac{\lambda_{t-1}^{1}}{2} \epsilon_{T-1}^{\prime}\right) u_{t-1}^{*}=2 u_{t-1}^{*} \Sigma_{t-1} u_{t-1}^{*} .
\end{aligned}
$$

Thus,

$$
\begin{aligned}
V_{t-1}^{*}\left(x_{t-1}, l_{t-1}\right)= & -u_{t-1}^{*}{ }^{\prime} \Sigma_{t-1} u_{t-1}^{*} \\
& +\left[\operatorname{Var}\left(Q_{t-1}\right)+\bar{\beta}_{2, t} E\left(Q_{t-1}^{2}\right)\right] l_{t-1}^{2} \\
& +\left[\lambda_{t-1} E\left(Q_{t-1}\right)+\bar{\beta}_{1, t} E\left(Q_{t-1}\right)\right] l_{t-1} \\
& -\lambda_{t-1}^{1} s_{t-1} x_{t-1}-\sum_{i=t}^{T-1} \frac{\left(\lambda_{i}^{1}\right)^{2}}{4} M_{i} \\
= & \bar{\beta}_{2, t-1} l_{t-1}^{2}+\bar{\beta}_{1, t-1} l_{t-1} \\
& -\lambda_{t-1}^{1} s_{t-1} x_{t-1}-\sum_{i=t-1}^{T-1} \frac{\left(\lambda_{i}^{1}\right)^{2}}{4} M_{i},
\end{aligned}
$$

where

$$
\begin{aligned}
& \bar{\beta}_{1, t-1}=\left(\lambda_{t-1}+\bar{\beta}_{1, t}\right) E\left(Q_{t-1}\right)-\lambda_{t-1}^{1} \epsilon_{T-1}^{\prime}\left(\Sigma_{t-1}\right)^{-1} \varphi_{t-1} \\
& \bar{\beta}_{2, t-1}=\operatorname{Var}\left(Q_{t-1}\right)+\bar{\beta}_{2, t} E\left(Q_{t-1}^{2}\right)-\varphi_{T-1}^{\prime}\left(\Sigma_{t-1}\right)^{-1} \varphi_{t-1}
\end{aligned}
$$

\section{Acknowledgments}

This work was supported by the National Natural Science Foundation of China (71101099, 11171237, 11101069), and by the Construction Foundation of Southwest University for Nationalities for the subject of Applied Economics (2011XWD-S0202).

\section{References}

[1] H. Markowitz, "Portfolio selection," Journal of Finance, vol. 7, pp. 77-91, 1952.

[2] D. Li and W.-L. Ng, "Optimal dynamic portfolio selection: multiperiod mean-variance formulation," Mathematical Finance, vol. 10, no. 3, pp. 387-406, 2000.

[3] X. Y. Zhou and D. Li, "Continuous-time mean-variance portfolio selection: a stochastic LQ framework," Applied Mathematics and Optimization, vol. 42, no. 1, pp. 19-33, 2000.

[4] A. Kell and H. Muller, "Efficient portfolios in the asset liability context," Astin Bulletin, vol. 25, pp. 33-48, 1995.

[5] W. F. Sharpe and L. G. Tint, "Liabilities-a new approach," Journal of Portfolio Management, vol. 16, pp. 5-10, 1990.

[6] M. Leippold, F. Trojani, and P. Vanini, "A geometric approach to multiperiod mean variance optimization of assets and liabilities," Journal of Economic Dynamics \& Control, vol. 28, no. 6, pp. 1079-1113, 2004.

[7] M. Leippold, F. Trojani, and P. Vanini, "Multiperiod meanvariance efficient portfolios with endogenous liabilities," Quantitative Finance, vol. 11, no. 10, pp. 1535-1546, 2011.

[8] M. C. Chiu and D. Li, "Asset and liability management under a continuous-time mean-variance optimization framework," Insurance, vol. 39, no. 3, pp. 330-355, 2006.

[9] S. X. Xie, Z. F. Li, and S. Y. Wang, "Continuous-time portfolio selection with liability: mean-variance model and stochastic LQ approach," Insurance, vol. 42, no. 3, pp. 943-953, 2008. 
[10] S. X. Xie, "Continuous-time mean-variance portfolio selection with liability and regime switching," Insurance: Mathematics \& Economics, vol. 45, no. 1, pp. 148-155, 2009.

[11] Y. Zeng and Z. Li, "Asset-liability management under benchmark and mean-variance criteria in a jump diffusion market," Journal of Systems Science \& Complexity, vol. 24, no. 2, pp. 317327, 2011.

[12] R. Strotz, "Myopia and inconsistency in dynamic utility maximization," Review of Economic Studies, vol. 23, pp. 165-180, 1955.

[13] S. Basak and G. Chabakauri, "Dynamic mean-variance asset allocation," Review of Financial Studies, vol. 23, pp. 2970-3016, 2010.

[14] J. Wang and P. A. Forsyth, "Continuous time mean variance asset allocation: a time-consistent strategy," European Journal of Operational Research, vol. 209, no. 2, pp. 184-201, 2011.

[15] X. Y. Cui, D. Li, S. Y. Wang, and S. Zhu, "Better than dynamic mean-variance: time inconsistency and free cash flow stream," Mathematical Finance, vol. 22, no. 2, pp. 346-378, 2012.

[16] Z.-P. Chen, G. Li, and J.-E. Guo, "Optimal investment policy in the time consistent mean-variance formulation," Insurance, vol. 52, no. 2, pp. 145-156, 2013.

[17] C. G. Li, Z. F. Li, K. Fu, and H. Q. Song, "Time-consistent optimal portfolio strategy for asset-liability management under mean-variance criterion," Accounting and Finance Research, vol. 2, pp. 89-104, 2013. 


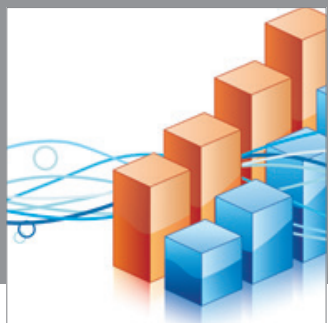

Advances in

Operations Research

mansans

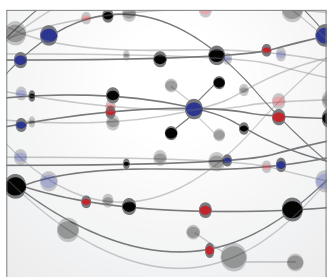

The Scientific World Journal
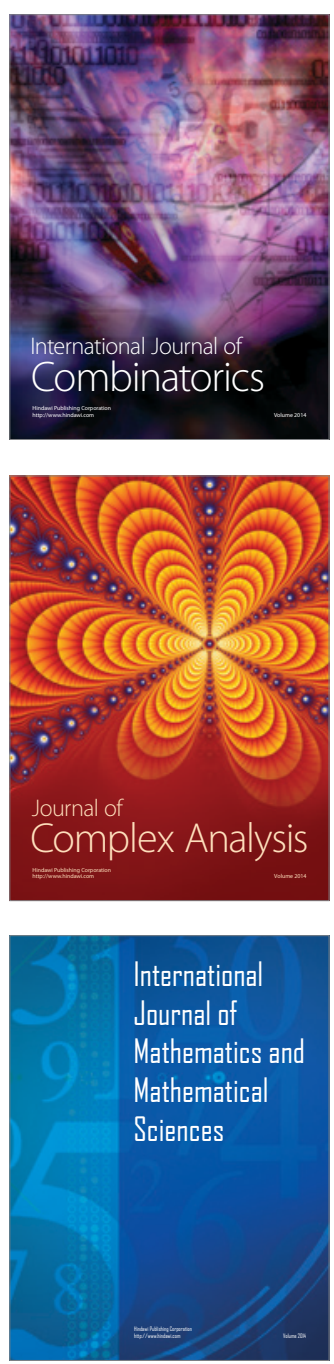
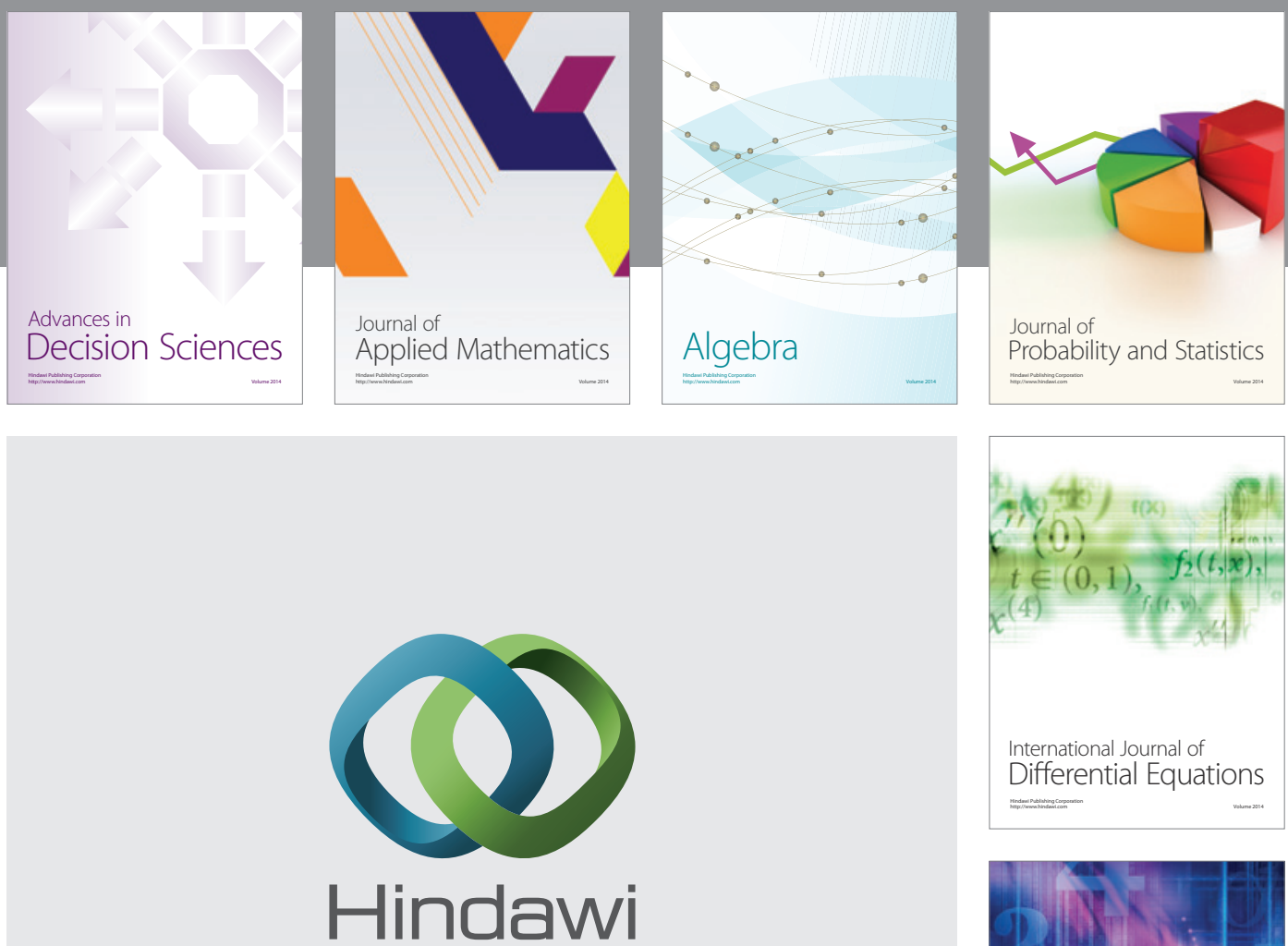

Submit your manuscripts at http://www.hindawi.com
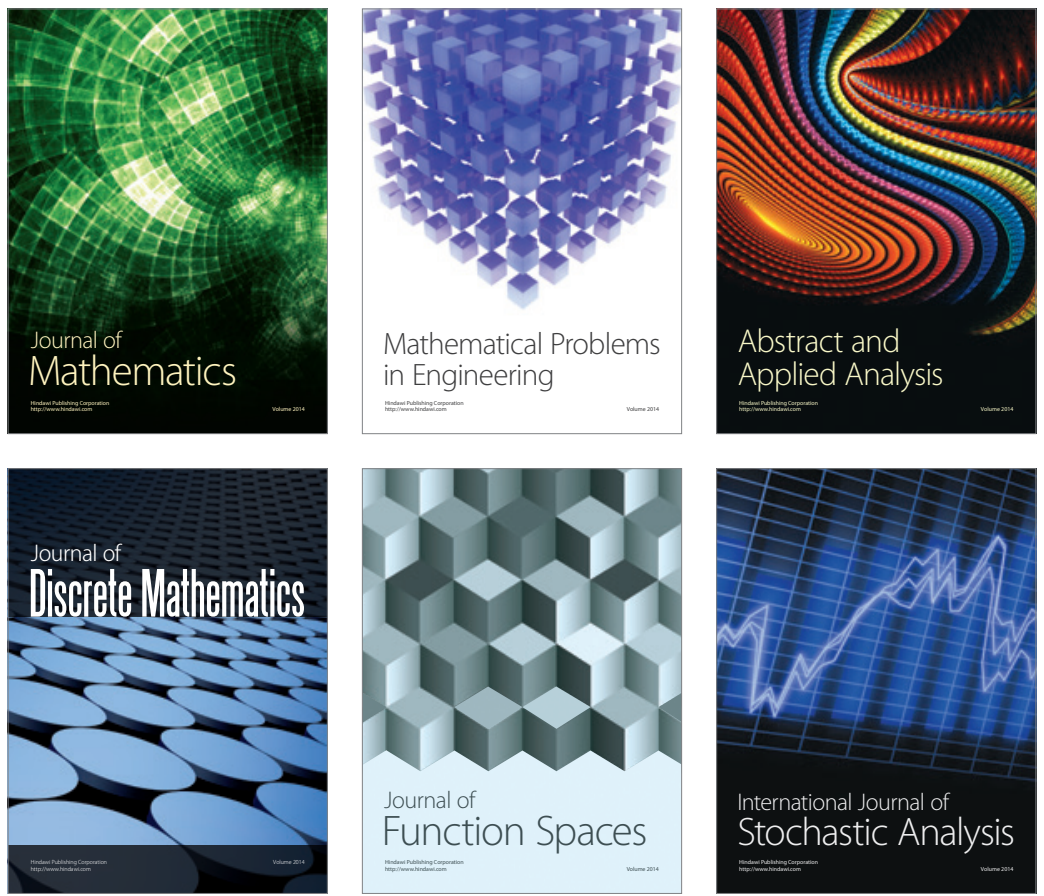

Journal of

Function Spaces

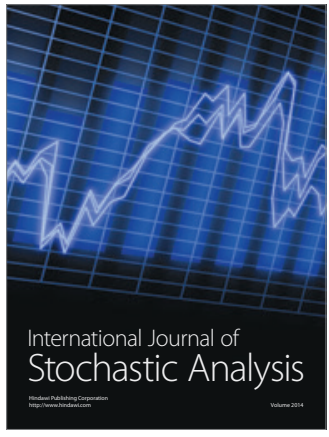

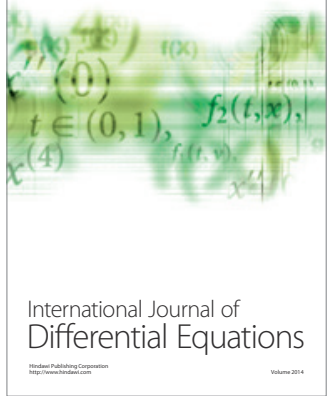
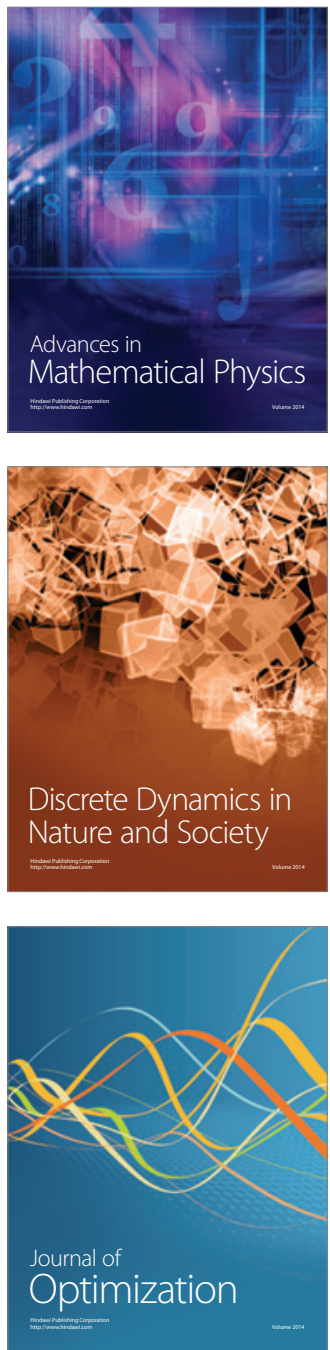